\title{
Nanostructure Selectivity for Molecular Adsorption and Separation: the Case of Graphyne Layers
}

Yusuf Bramastya Apriliyanto, ${ }^{\dagger, \ddagger}$ Noelia Faginas Lago, ${ }^{*}, \dagger, \| \circledast$ Andrea Lombardi, ${ }^{\dagger, \|}$ Stefano Evangelisti, ${ }^{\ddagger}$ Massimiliano Bartolomei, ${ }^{\S}$ Thierry Leininger, ${ }^{\ddagger}{ }^{\circ}$ and Fernando Pirani ${ }^{\dagger}$

${ }^{\dagger}$ Dipartimento di Chimica, Biologia e Biotecnologie, Università degli Studi di Perugia, Via Elce di Sotto 8, 06123 Perugia, Italy

${ }^{\ddagger}$ Laboratoire de Chimie et Physique Quantiques, IRSAMC, Université de Toulouse III-Paul Sabatier, 118 Route de Narbonne, 31062 Toulouse Cedex, France

${ }^{\S}$ Instituto de Física Fundamental, Consejo Superior de Investigaciones Científicas (IFF-CSIC), Serrano 123, 28006 Madrid, Spain

"Consortium for Computational Molecular and Materials Sciences (CMS) ${ }^{2}$, Via Elce di Sotto, 8, 06123 Perugia, Italy

Supporting Information

ABSTRACT: Graphynes are porous derivatives of graphene that can be considered as ideal $2 \mathrm{D}$ nanofilters. Here, we investigate by theoretical methods graphtriyne multilayers, proposing them as membranes featuring pores of subnanometer size suitable for $\mathrm{CO}_{2} / \mathrm{N}_{2}$ separation and $\mathrm{CO}_{2}$ uptake. The potential energy surfaces, representing the intermolecular interactions within the $\mathrm{CO}_{2} / \mathrm{N}_{2}$ gaseous mixtures and between the graphtriyne layers and the molecules, have been formulated in an internally consistent way, by adopting potential models far more accurate than the traditional Lennard-Jones functions, routinely used to predict static and dynamical properties of matter. The new force fields so obtained and tested on accurate ab initio calculations have been used to perform extensive molecular dynamics simulations of membrane selectivity and adsorption. The accuracy of the potentials granted a quantitative description of the interactions and realistic results for the dynamics under a wide range of conditions of applied interest, indicating a single-layer permeation ratio $\mathrm{CO}_{2} / \mathrm{N}_{2}$ of 4.25 (meaning that permeations of $\mathrm{CO}_{2}$ are typically 4.25

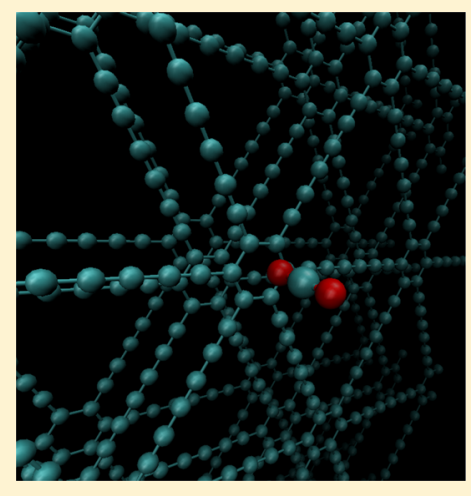
times those of $\mathrm{N}_{2}$ ). At low pressure, graphtriyne bilayer membranes exhibit good performances as a molecular sieving candidate for postcombustion $\mathrm{CO}_{2}$ separation because of a high permeance and a relatively good selectivity. On the other hand, graphtriyne trilayer membranes present a relatively high interlayer adsorption selectivity and a high $\mathrm{CO}_{2}$ uptake. Such properties make these graphyne nanostructures versatile materials competitive with other carbonbased adsorbing membranes suitable to cope with post-combustion $\mathrm{CO}_{2}$ emissions. Moreover, guidelines for the extension of the proposed methodology to carbon nanostructures and other gaseous mixtures of relevance for atmosphere and combustion are also provided.

\section{INTRODUCTION}

The investigation of chemical and physical processes involving nanostructures makes it possible today the discovery of new electric and magnetic phenomena (see, for instance, refs $^{1-3}$ ) of interest in several fields of fundamental and applied research.

A current issue of great relevance is the control and reduction of the amount of $\mathrm{CO}_{2}$ released into the atmosphere. This has increased significantly in recent decades, ${ }^{4,5}$ mostly because of the excess of its emission originated by fossil fuel combustion, leading to unavoidable environmental pollution issues that are indeed strongly connected to the global warming phenomenon. ${ }^{5} \mathrm{New}$ technologies for the efficient carbon capture in postcombustion mixtures are therefore highly needed to control the $\mathrm{CO}_{2}$ emissions at a level that could minimize their impact on the environment. Novel porous materials are widely considered as a promising media for the efficient capture of $\mathrm{CO}_{2}$ within their pores or by adsorption upon their surfaces. As a matter of fact, several studies involving a variety of materials, starting from porous carbons, zeolites, metal-organic frameworks (MOFs), polymers, and even a slurry concept (solid adsorbents in a liquid absorbent), have been reported in the last years. ${ }^{6-12}$

A possible alternative to porous adsorbing materials is represented by the use of membranes as molecular sieves, as shown in recent experimental ${ }^{13}$ and theoretical ${ }^{14}$ studies. Although they have a main issue about a trade-off between selectivity and permeability, their simplicity, efficiency, and lowimplementation cost make membrane-based technologies appealing for gas separation. ${ }^{15-18}$ Among other materials, carbon-based membranes attracted much attention because of their properties: they are chemically inert and exhibit some advantages such as good thermal stability and hydrophobicity. ${ }^{19}$ Therefore, it seems convenient to propose them

Received: May 24, 2018

Revised: June 21, 2018

Published: June 21, 2018 
for $\mathrm{CO}_{2}$ separation in postcombustion mixtures, where humid and hot gas streams have to be treated.

Graphene is a single atomic layer belonging to the class of carbon allotropes. It is one of the thinnest materials whose properties can be modulated through functionalization, which can be in principle obtained by binding different groups or by introducing holes. For these reasons, its porous derivatives are currently considered ${ }^{20,21}$ as promising platforms for the design of carbon-based membranes suitable for gas separation and other applications, for example, water purification $^{22}$ and thermal conduction. ${ }^{23}$

An important drawback of graphene is its tendency to capture gas-phase molecules, forming stable physisorption aggregates, because of strong long-range dispersion forces, arising from the high number of carbon atoms per unit surface on the external layer. This tendency must be combined with a strong repulsion at a short range (because of the small size of the hexagonal opening compared to the molecular size), which hinders gas intercalation and limits the gas-uptake capacity.

A valid alternative to porous graphene-based carbon materials is represented by a class of compounds called $\gamma$ graphynes. They are new $2 \mathrm{D}$ carbon allotropes formed by an alternation of $\mathrm{sp}-\mathrm{sp}^{2}$ hybridized carbon atoms, first predicted $^{24}$ in the late-1980s and then recently synthesized. ${ }^{25}$ The $\gamma$-graphynes exhibit similar properties with respect to graphene but with triangle-like pores of subnanometer dimensions that are uniformly distributed and adjustable in size. $^{25}$ A very recent study ${ }^{26}$ reported that $\gamma$-graphynes, in particular graphtriyne ("tri" indicates that each phenyl ring is connected to each of the six others through chains composed by three conjugated acetylenic bonds placed as bridges), show a strong and preferential physisorption of $\mathrm{CO}_{2}$ molecules within its pores with respect to other species of atmospheric interest such as $\mathrm{N}_{2}$ and $\mathrm{H}_{2} \mathrm{O}$. However, the collection of a realistic phenomenology about the selective properties of $\gamma$ graphynes requires extensive theoretical studies of the membrane-gas dynamics based on a proper formulation of the force fields, as a condition to obtain reliable results from molecular dynamics (MD) simulations.

In this paper, we present an integrated (semiempirical modeling-ab initio calculations) approach best suited to describe interactions and dynamics in gas-membrane systems and apply it to assess the properties of $\gamma$-graphyne membranes as molecular sieves for $\mathrm{CO}_{2} / \mathrm{N}_{2}$ gaseous mixtures. The approach features accurate potential energy surfaces (PESs) providing in a proper and internally consistent way the intermolecular interactions occurring both in the $\mathrm{CO}_{2} / \mathrm{N}_{2}$ gaseous mixtures and between the gas-phase molecules and the membranes. Extensive MD simulations are exploited to characterize the performance of graphtriyne-based membranes for the $\mathrm{CO}_{2} / \mathrm{N}_{2}$ separation and the $\mathrm{CO}_{2}$ uptake, considering both single and multilayer membranes.

\section{METHODS}

As stressed in the previous section, to perform reliable and realistic MD simulations, a key requirement is represented by the use of accurate analytic force fields, reproducing quantitatively the intermolecular interactions within symmetric and asymmetric pairs in gas phase, formed by the $\mathrm{CO}_{2}$ and $\mathrm{N}_{2}$ molecules, and those occurring between the molecules and the carbon nanostructure. All MD simulations have been carried out by using the DL_POLY program, whereas the intermolecular potential energy $\mathrm{V}$ has been defined in terms of a potential function introduced in our laboratory ${ }^{27}$ from the analysis of an ample phenomenology observed on prototypical systems and called improved Lennard-Jones (ILJ) model. The proposed force fields, describing different systems in an internally consistent way, have been tested and improved in their formulation by exploiting their comparison with results from high-level $\mathrm{ab}$ initio calculations.

Modeling of the Interactions and Force Fields. As for the parameterization of the force fields, in our model, the total potential energy, denoted by $V_{\text {tot }}$, represents the interaction between any interacting pair involving the $\mathrm{CO}_{2}$ and $\mathrm{N}_{2}$ molecules in gas phase and the $\gamma$-graphyne membrane. $V_{\text {tot }}$ is given by the sum of two terms, upon the assumption that the electrostatic interactions, denoted by $V_{\mathrm{el}}$, and the nonelectrostatic ones, denoted by $V_{\text {nel, }}$ are separable. In our model, $V_{\mathrm{el}}$ arises from the anisotropic charge distributions that control on each partner the permanent electric multipole value, whereas $V_{\text {nel }}$ accounts for size (or Pauli) repulsion, prevalent at a short range, and dispersion and induction attraction, dominant at a long range.

The electrostatic contribution is easily evaluated through the Coulomb law assigning to the molecules point-of-charge distributions compatible with the molecular electric quadrupole moment, see Figure S1 in the Supporting Information. The graphtriyne layers are considered to be neutral and apolar; therefore the $V_{\mathrm{el}}$ term was included only in $\mathrm{CO}_{2}-\mathrm{N}_{2}, \mathrm{CO}_{2}-$ $\mathrm{CO}_{2}$, and $\mathrm{N}_{2}-\mathrm{N}_{2}$ interactions. On the other hand, $V_{\text {nel }}$ is, in general, the interaction component more difficult to formulate because simple analytic representations, preferable to speed up the massive calculations required by simulations of large systems, present the drawback of a wrong description of the long-range tail and the repulsive wall of the potential-energy profiles.

In this investigation, we have exploited the ILJ formulation of the interaction to represent $V_{\text {nel, }}$ overcoming the inadequacies of the classical LJ model because of an excess of attraction at a long range and a too strong repulsion at a short range. ${ }^{27}$ This permits to obtain a suitable analytic formulation of $V_{\text {nel }}$ as a sum of pairwise ILJ potential energy functions, each one represented as ${ }^{27-29}$

$$
V_{\mathrm{ILJ}}=\varepsilon\left[\frac{m}{n(r)-m}\left(\frac{r_{0}}{r}\right)^{n(r)}-\frac{n(r)}{n(r)-m}\left(\frac{r_{0}}{r}\right)^{m}\right]
$$

where $n(r)$ is given by

$$
n(r)=\beta+4.0\left(\frac{r}{r_{0}}\right)^{2}
$$

and $m$ takes the value of 6 for interacting the neutral molecules, such as the present ones. $V_{\text {ILJ }}$ represents the nonelectrostatic contributions between two pairs of interaction centers placed on different molecules and separated by a distance $r$. The interaction centers are usually chosen to coincide with atoms (present case), middle point of bonds, or, in the case of larger molecules, centers of mass atom groups. The relevant parameters, $\varepsilon$ and $r_{0}$, representing the well depth and the equilibrium distance of the interacting pair, respectively, are conventionally determined by using the values of the polarizability associated with each interaction center. The advantages of the ILJ formulation over the traditional $\mathrm{LJ}$ form have been well-discussed elsewhere. ${ }^{30-33}$ Here, we remark that by modulating the $\beta$ parameter (usually for neutral systems $\beta=$ 
$8.5 \pm 2.5),{ }^{31}$ the variations in $V_{\mathrm{el}}$ coming from modifications of the charge distribution can be compensated and the role of the other less-important contributions indirectly included.

The zero order $r_{0}, \varepsilon$, and $\beta$ parameters used in the resulting PES have been predicted by the effective polarizability values of the atoms in the gas-phase molecules and in the graphtriyne layer. Then, they have been fine-tuned, exploiting the comparison of the predicted interaction with results from accurate $a b$ initio computations ${ }^{26,34-40}$ obtained at the MP2C level of theory, ${ }^{41}$ best-suited for the intermolecular interactions characterizing the studied systems. For the total potential energy electronic structure calculations, the aug-cc-pVTZ and aug-cc-pVQZ Dunning basis sets ${ }^{42}$ were adopted for the pore prototype and the $\mathrm{CO}_{2}\left(\mathrm{~N}_{2}\right)$ molecule, respectively, in the same spirit of the work in ref 26 . The obtained energy profiles, properly corrected for the basis set superposition energy, correspond to monomers considered as rigid bodies. The reported calculations have been performed by using the MOLPRO quantum-chemistry package. ${ }^{43,44}$

MD Simulations. All of the MD simulations have been performed in the canonical (NVT) ensemble with periodic boundary conditions (PBC) in all directions, using the DL POLY program. ${ }^{45}$ The size of the simulation box was of $36 . \overline{1} \times 62.52 \times 140.00 \AA^{3}$. To enhance the statistical accuracy of the data collected, ${ }^{17}$ the box actually contained two parallel membranes with dimensions $36.11 \times 62.52 \AA^{2}$ that were placed in the middle and at the end of the box. A NoseHoover thermostat with a relaxation constant of 0.5 ps has been employed to keep the temperature $T$ of the system at 300 $\mathrm{K}$. The cutoff distance for the nonelectrostatic and electrostatic components has been set to $15 \AA$, and the Ewald method has been applied for the calculation of electrostatic contributions. During the simulations, the graphtriyne membrane has been considered as a frozen structure and the gas molecules treated as rigid bodies. Each simulation has been performed for $5 \mathrm{~ns}$ after a properly long equilibration period with a fixed time step of $1 \mathrm{fs}$, and the data have been collected at every 2 ps. After each production run, the trajectories have been recorded and the results analyzed.

The temperatures in the simulations had relative fluctuations ranging from 20 to $3 \%$ (relative standard deviations from $\sim 7$ to $1 \%)$ passing from lower to higher pressures.

\section{RESULTS AND DISCUSSION}

The energy profiles characterizing the interactions in gaseous mixtures have been shown and extensively discussed in previous works, ${ }^{34,35,38}$ whereas cuts of the PESs, describing the $\mathrm{CO}_{2}-$ and $\mathrm{N}_{2}$-graphtriyne interactions for specific orientations of the molecules, are given in Figures 1 and 2, where a very good agreement can be observed between the analytic force fields and the accurate ab initio estimations. Such a remarkable matching has been considered an important condition to obtain reliable PESs in the complete relative configuration space.

At this point, it is relevant to stress two basic characteristics of the adopted force field formulation, concerning its reliability and its easy extension/generalization to different molecular systems:

(i) The standard Lennard-Jones potential model, still widely used in MD simulations, is hardly capable to correctly describe the main features of the $\mathrm{CO}_{2}\left(\mathrm{~N}_{2}\right)$-graphtriyne interaction. This is exemplified in Figures S2 and S3 in

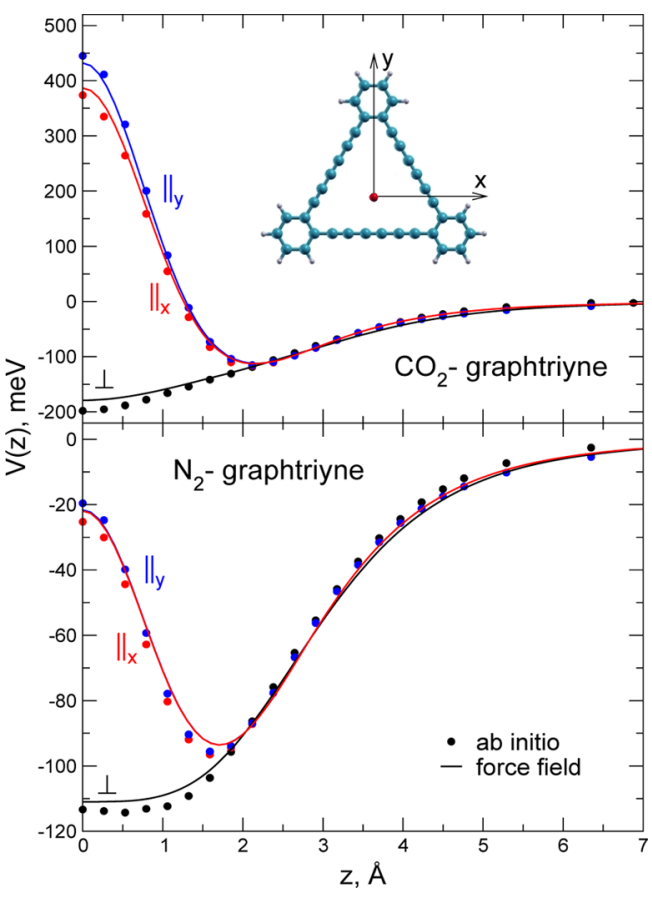

Figure 1. Energy profiles for the interaction between a molecular prototype of the graphtriyne pore (octadecadehydrotribenzo ${ }^{26}$ annulene, see inset in the upper panel) and the $\mathrm{CO}_{2}$ (top panel) and $\mathrm{N}_{2}$ (bottom panel) molecules. The reported curves refer to the vertical approach of the $\mathrm{CO}_{2}\left(\mathrm{~N}_{2}\right)$ center of mass toward the geometric center of the pore. Two parallel (the molecular axis oriented along the $x$ and $y$ directions) and one perpendicular (the molecular axis oriented along the $z$ direction) geometries of the $\mathrm{CO}_{2}\left(\mathrm{~N}_{2}\right)$ molecules have been considered. Full circles and solid lines correspond to the profiles as obtained from MP2C ab initio calculations [using aug-cc-pVXZ basis sets $(X=T, Q)$ ] and the analytic force field, respectively.

the Supporting Information, where the predictions obtained by the AMBER general force field, ${ }^{46}$ compared to the accurate $a b$ initio results, show strong deviations, for example, dramatically overestimating the energy barriers encountered by the $\mathrm{CO}_{2}$ molecules crossing the membrane;

(ii) The potential parameters involved in the ILJ formulation, having a defined physical meaning, exhibit a high portability degree and an easy transfer when passing from a molecular system to another. The high portability comes from the existence of scaling laws (correlation formulas) connecting the ILJ potential parameters $\varepsilon$ and $r_{0}$ (see eq 2 in the Methods section), with effective atomic, bond, and molecular polarizabilities (see ref 27 and additional references given in the Supporting Information). Therefore, the modification of the potential, adapting to a different mixture or carbon nanostructure, is straightforward because good estimates of the new parameters are immediately obtained from the change in the involved components of the polarizability. Molecules or surfaces at different complexity can be treated and modeled within the same scheme, by properly partitioning them into functional groups, and/or chemical bonds and/or pseudoatomic interaction centers, assigning to each one a proper value of the polarizability component from which the parameters are easily obtained. Although to provide complete sets of 


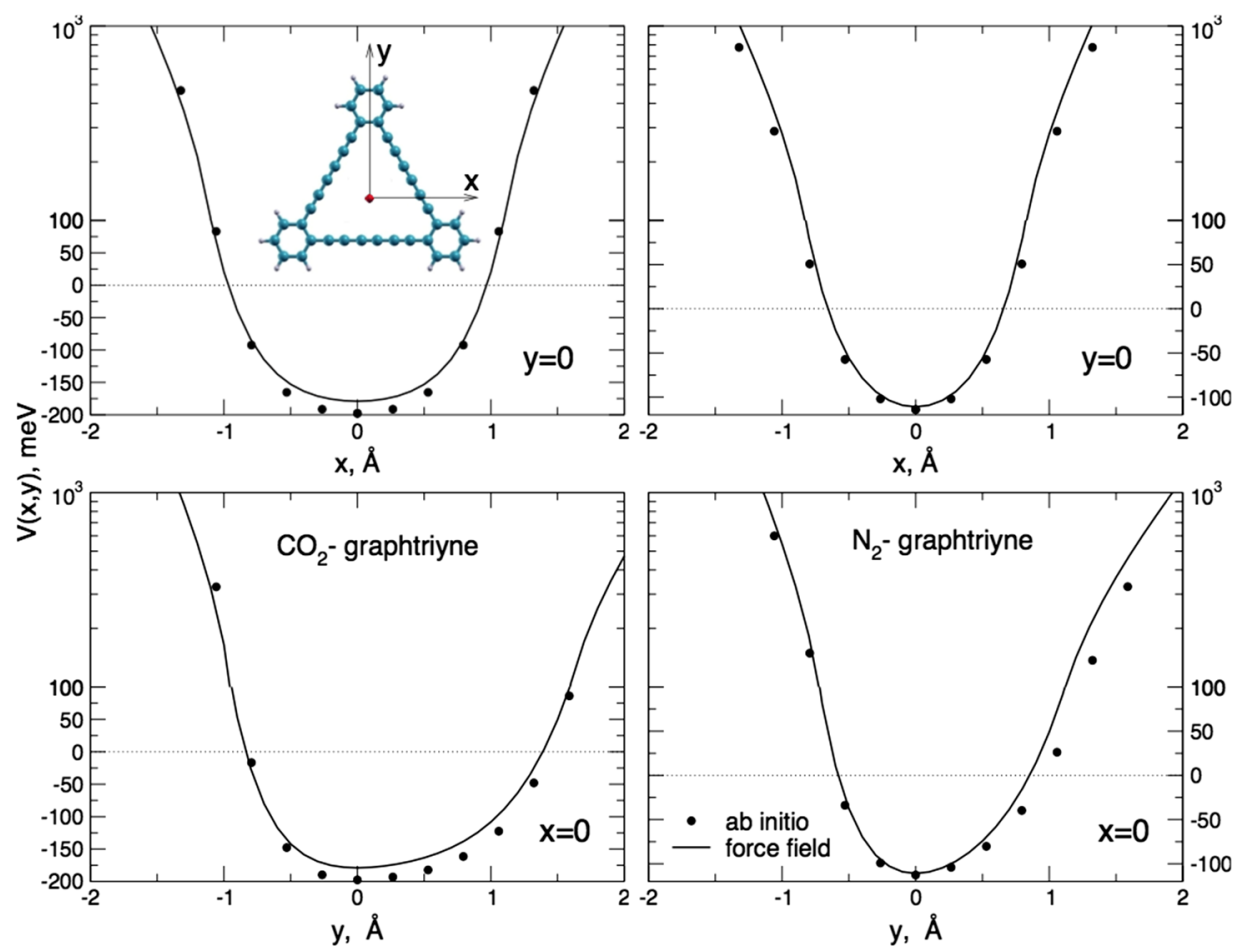

Figure 2. In-plane $(z=0)$ behavior of the interaction between the graphtriyne pore and $\mathrm{CO}_{2}\left(\mathrm{~N}_{2}\right)$, left (right) panel, as a function of the molecule center of mass displacements (along $x$ and $y$ directions) from the geometric center of the pore. The $\mathrm{CO}_{2}\left(\mathrm{~N}_{2}\right)$-graphtriyne geometry is such that the molecular axis is perpendicular to the pore plane. Full circles and solid lines correspond to the profiles as obtained from MP2C ab initio calculations [using aug-cc-pVXZ basis sets $(\mathrm{X}=\mathrm{T}, \mathrm{Q})]$ and the analytic force fields, respectively.

parameters for different systems is beyond the purpose of this work, in the Supporting Information, we shortly report the extension of the present van der Waals (vdW) potential formulation to the case of graphdiyne$\mathrm{CO}_{2}\left(\mathrm{~N}_{2}\right)$ interactions, exploiting the variation of the pore structure, see Figure S4 in the Supporting Information for a representation of the potential energy profiles for the graphdiyne- $\mathrm{CO}_{2}\left(\mathrm{~N}_{2}\right)$ interactions in the limiting configurations. Moreover, as a further example of portability/extension of the methodology, we also estimated the potential parameters necessary to carry out MD simulations of gaseous mixtures including $\mathrm{O}_{2}$ as additional species. All of the $\mathrm{O}_{2}$ and $\mathrm{N}_{2}$ potential parameters appear very similar, as confirmed by several experimental and theoretical findings because the value of the effective $\mathrm{O}$ atomic polarizability $\left(0.8 \mathrm{~A}^{3}\right)$ in $\mathrm{O}_{2}$ is close to that of the $\mathrm{N}$ atom $\left(0.9 \mathrm{~A}^{3}\right)$ in $\mathrm{N}_{2}$. For the complete sets of data see the Supporting Information, where guidelines useful to formulate a force field for $\mathrm{H}_{2} \mathrm{O}$ - and $\mathrm{C}_{2} \mathrm{H}_{2}$-graphtriyne systems are also given.

Therefore, in the proposed methodology, the relationship between the potential parameters and the molecular polarizability components is a basic way to connect the vdW interaction to a physically meaningful quantity and to obtain internally consistent force fields in systems involving molecules of different complexity. The features of the $\mathrm{CO}_{2}-\mathrm{CO}_{2}, \mathrm{CO}_{2}-$ $\mathrm{N}_{2}$, and $\mathrm{N}_{2}-\mathrm{N}_{2}$ PESs, discussed in detail in refs, ${ }^{34,35,38,47}$ clearly show that the $\mathrm{CO}_{2}-\mathrm{CO}_{2}$ interaction is stronger and more anisotropic. Such features, combined with those shown in Figures 1 and 2, can be exploited to attempt a preliminary rationalization of possible selectivity effects arising from the different strength and anisotropy of the interactions involved. In particular, although the molecule-molecule PESs affect the collision dynamics in gas phase, control the relative molecular motion, and the possible molecular packing within each pore, the molecule-graphtriyne interaction controls the probability of formation and the stability of the physisorption state that subsequently can induce the molecular passage through each layer channel. Results in Figures 1 and 2 suggest that adsorption and permeation of $\mathrm{CO}_{2}$ on graphtriyne layers must be favored with respect to $\mathrm{N}_{2}$, being the long-range attraction stronger and the related potential well much deeper and wider. Moreover, at the center of the pore $(z=0)$, although $\mathrm{N}_{2}$ undergoes an attraction in both parallel and perpendicular approach geometries, $\mathrm{CO}_{2}$ is strongly attracted when confined in the perpendicular geometry but also largely repelled in parallel configurations. This clearly indicates that the adsorption phenomenon is expected to be strongly stereoselective with respect to the $\mathrm{CO}_{2}$ molecular orientation and much less sensitive to the orientation of $\mathrm{N}_{2}$. The parameters used in the force fields are reported in Tables S1 and $S 2$ in the Supporting Information.

As for the electrostatic $V_{\mathrm{el}}$ term, three-charge-site $\mathrm{s}^{34,38}$ and five-charge-site ${ }^{35}$ distributions have been adopted for $\mathrm{N}_{2}$ and $\mathrm{CO}_{2}$, respectively, to reproduce also the corresponding molecular quadrupole moments. A massless point charge in the middle of the $\mathrm{N}-\mathrm{N}$ and the $\mathrm{C}-\mathrm{O}$ bonds has been inserted, ${ }^{34,38}$ as illustrated in Figure $\mathrm{S} 1$ of the Supporting Information.

Three different membranes (see Figure 3) were considered, corresponding to graphtriyne single, bi-, and trilayers. The structures illustrated in the figure are those obtained in ref 26 as optimized configurations determined through proper periodic density functional theory calculations. It has to be stressed that although the pore diameter can be determined to be about $6.9 \AA$ from geometric considerations, its effective size 

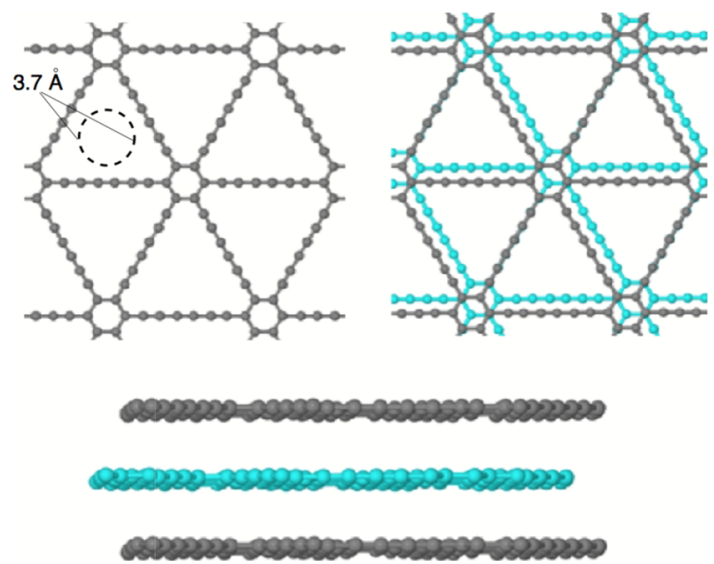

Figure 3. Three types of membranes used in the simulations (graphtriyne single, bi-, and trilayer). The dashed circle depicted inside the triangular pore roughly corresponds to its effective available (free) area whose diameter is about $3.7 \AA$.

is actually quite lower because of the vdW size of the surrounding carbon atoms. As a matter of fact, by considering the vdW radius of the acetylenic carbon bonds $(\sim 1.6 \AA)$, the effective available (free) area of the pore is equivalent to that of a circle with a diameter of $3.7 \AA$, represented in Figure 3.

In the case of the bilayer membrane, one of the carbon sheets is parallel to the other and shifted to $1.55 \AA$ with respect to the AA-type stacking. Depending on the two main directions of the parallel displacement, two different structures, namely $A B-1$ and $A B-2$, were identified in ref 26 (see Figure 1 in there) and found to lead to practically coincident energy minima. For the sake of simplicity, in the present work, we only considered the AB-1 structure (see Figure 3), for which the layer shift is along one of the polyacetylenic chains. Analogously, for the trilayer membrane, we considered the AB1 parallel arrangement and, in all cases, the interlayer separation was given the optimized value of $3.45 \AA$.

To our knowledge, graphtriyne layers have not been synthesized yet, but recent advances in the obtention of graphdiyne nanowalls ${ }^{48}$ may suggest that an analogous approach could also be employed for other $\gamma$--graphynes with pores of larger size.

In MD simulations, three different gaseous systems (pure $\mathrm{N}_{2}$, pure $\mathrm{CO}_{2}$, and a mixture with equal fractions of $\mathrm{N}_{2}$ and $\mathrm{CO}_{2}$ ) interacting with the membranes were investigated. For each system, eight different amounts of gas were loaded into the box to study the influence of different initial pressures. The gas pressure for the NVT simulations at $300 \mathrm{~K}$ was obtained using the Peng-Robinson equation of state ${ }^{49}$ (see Tables S3 and S4 in the Supporting Information). In most of the simulations, the gas molecules were equally distributed into the two regions of the box at random positions; therefore, no pressure gradient was applied inside the box. During each simulation, the gas molecules could cross the membranes many times in both directions. The number of permeating molecules was then monitored along with the $z$-density profile (density along the $z$ direction, normal to the plane of the membranes). To obtain converged values of the permeance, avoiding exceedingly long simulation times, the number of molecules in the box should not be too low. Figure 4 shows snapshots of the simulations of the $\mathrm{CO}_{2} / \mathrm{N}_{2}$ mixture with single (left panel) and trilayer (right panel) membranes.

Single-Layer Membrane. Initially, simulations were performed by considering a single-layer membrane and pure $\mathrm{CO}_{2}$, distributing the gas molecules in one of the two regions of the box, separated by the membrane. At the beginning of each calculation, an equilibration run was performed, for a properly long simulation time (a few nanosecond) to achieve a configuration representative of the equilibrium state. The configurations obtained after the equilibration runs showed that the gas molecules were distributed almost equally in both regions of the box, consistently to what was expected for the final concentration of the molecules that should be the same in such two regions of the box. The equilibration times were found to be considerably longer for the simulations at the higher pressure values because of the larger number of molecules in the box.

Accordingly, runs performed for the simulation of the single layer with pure $\mathrm{CO}_{2}$ and $\mathrm{N}_{2}$ gases were started as a continuation of the equilibration trajectories with the molecules equally distributed into two regions of the simulation box. During the simulations, the permeation events occurring in both directions and for each of the membranes were counted. Figure 5 shows the average total numbers of permeation events of the pure $\mathrm{CO}_{2}$ and $\mathrm{N}_{2}$ gas molecules as a function of the simulation time (various simulations were performed, for each pressure value). The gas permeation rate can be estimated as the slope of the function that linearly fits the plotted points, also represented in Figure 5 (red lines). By this approach, ${ }^{14}$ we obtained permeation rates for $\mathrm{CO}_{2}$ and $\mathrm{N}_{2}$ of about 0.81 and 0.17 molecules $\mathrm{ps}^{-1}$, respectively. This different behavior of the permeation rates is consistent with the attractive forces characterizing the potential wells whose minimum is at the center of the membrane pore, where penetration occurs. The gradient of the potential is always much larger for $\mathrm{CO}_{2}$ and the attraction is much stronger, as it can be seen in Figure 1 (potential as a function of the distance $z$ from the plane) and Figure 2 (potential as a function of the position on the membrane plane). Moreover, it can be seen that the potential wells for the interactions with $\mathrm{CO}_{2}$ are wider than those of $\mathrm{N}_{2}$, meaning a longer range for the attraction. These features of the potentials explain the higher permeation rates of $\mathrm{CO}_{2}$ even in mixtures (see also Figure 9 below).
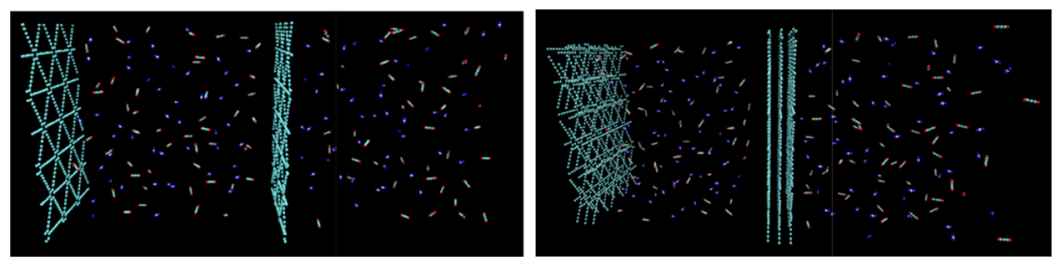

Figure 4. Snapshots of the simulation box for mono- and trilayer membranes with a mixture of $\mathrm{CO}_{2}$ (red and gray molecules in the figure) and $\mathrm{N}_{2}$ (blue molecules in the figure). 

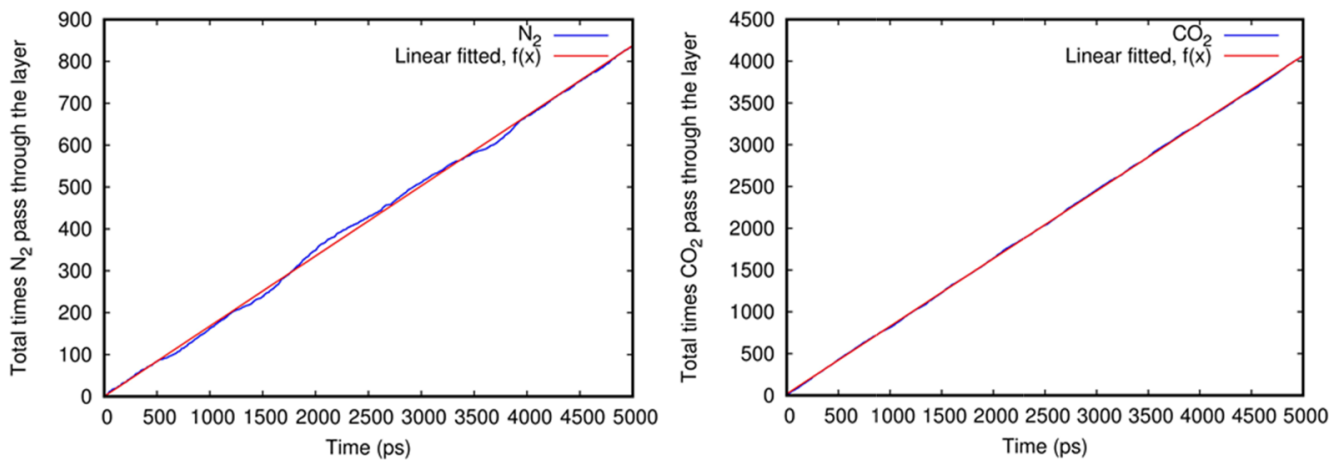

Figure 5. Average total permeation events vs the simulation time for $\mathrm{N}_{2}$ at a pressure of $17.99 \mathrm{~atm}$ (left panel) and $\mathrm{CO}_{2}$ at a pressure of 17.88 atm (right panel) crossing the single-layer membrane (blue lines). The red lines represent the linear fitting functions $f(x)$.
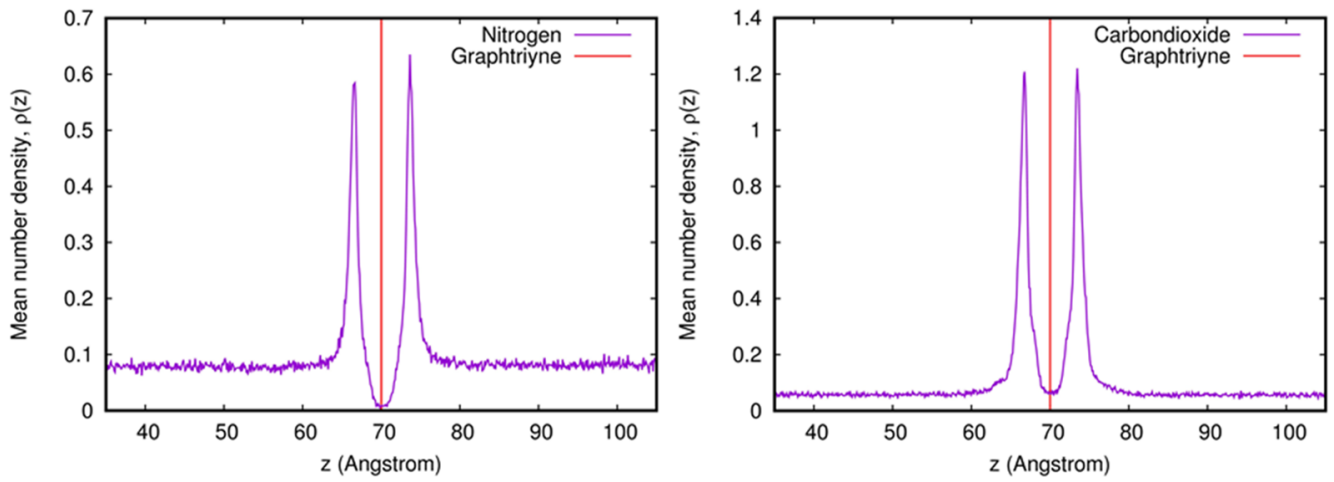

Figure 6. $z$-Density profile plotted as a function of the $z$-axis in the range $35-105 \AA$ for $\mathrm{N}_{2}$ at 17.99 atm (left panel) and $\mathrm{CO}_{2}$ at 17.88 atm (right panel). The single-layer membrane is located at $70 \AA$.

From Figure 5, it can also be seen that both $\mathrm{CO}_{2}$ and $\mathrm{N}_{2}$ can penetrate through the monolayer even when the simulation time is still below 500 ps. This is due to the fact that the pore size is large enough compared to the dimension of the considered molecules. More in detail, the estimated vdW radius, perpendicular to the molecular bond, is about $1.6 \AA$ for both molecules, whereas the radius parallel to the molecular axis is about 2.0 and $2.3 \AA$, for $\mathrm{N}_{2}$ and $\mathrm{CO}_{2}$, respectively. Moreover, as anticipated in the previous section and illustrated in Figures 1 and 2, no energy barriers are operative for penetration processes of both $\mathrm{CO}_{2}$ and $\mathrm{N}_{2}$ molecules approaching the pore center oriented perpendicularly. In particular, each potential energy profile exhibits a minimum right in the in-plane configuration (see Figures 1 and 2) that is exactly at the $z$-coordinate where the membrane layer is located. $^{26}$

The stronger $\mathrm{CO}_{2}$ attraction is also reflected by the $z$-density profiles $\rho(z)$ reported in Figure 6, where the mean number density along the coordinate $z$ indicates that the membrane exhibits a strong affinity for $\mathrm{CO}_{2}$. A close look at Figure 6 allows to notice that, although the simulation box is symmetric especially after the application of PBCs, the $\rho(z)$ profiles are not completely symmetric with respect to the membrane position at $70 \AA$. These small deviations, considering the symmetry of the simulation box and the PBCs enforced, can be attributed to mere statistical fluctuations.

Furthermore, it can be also observed that the $z$-density do not present a peak in correspondence with the membrane position, where, according to the potential-energy profiles for the vertical approach in the perpendicular orientation shown in Figure 1, there is a minimum of the potential energy. This result can be interpreted in terms of the oscillatory motion of the molecules in a physisorption state. In this state, molecules can either oscillate crossing the membrane, with a trajectory evolving over the black potential energy curve in Figure 1 (symmetric with respect to the membrane position) or remain for a while on one side, trapped in the potential wells represented by blue and red curves in Figure 1, until they get the optimal orientation for crossing. For $\mathrm{N}_{2}$, there are looser stereodynamic requirements because the energy barriers for crossing with orientations other than perpendicular (Figure 1 lower panel, red and blue curves) are smaller than those of $\mathrm{CO}_{2}$ (Figure 1 upper panel, red and blue curves). Consistently, the $z$-density peaks for $\mathrm{CO}_{2}$ appear slightly thicker. It is known that classical oscillating systems spend more time in the neighborhoods of turning points, where kinetic energy approaches to zero and less time in the neighborhood of the minimum energy point. This explains at least qualitatively the $\rho(z)$ profiles. In the present simulations at $300 \mathrm{~K}$, the thermal translational energy for a free molecule is about $40 \mathrm{meV}$ plus an energy transfer from rotations induced by the stereoselective character of the PES, tending to align the molecules preparing them for crossing. The positions of the classical turning points for such state of the molecules, under these conditions, are therefore qualitatively consistent with the positions of the $\rho(z)$ peaks at a distance of $\sim 3.0 \AA$ from the surface. This contrasts with the quantum oscillator case, where in the ground-state, the probability density has a peak at the center of the potential well. Performing classical MD simulations, peaks of the $z$-density can therefore be expected to arise (see Figure 6) at the turning points. 

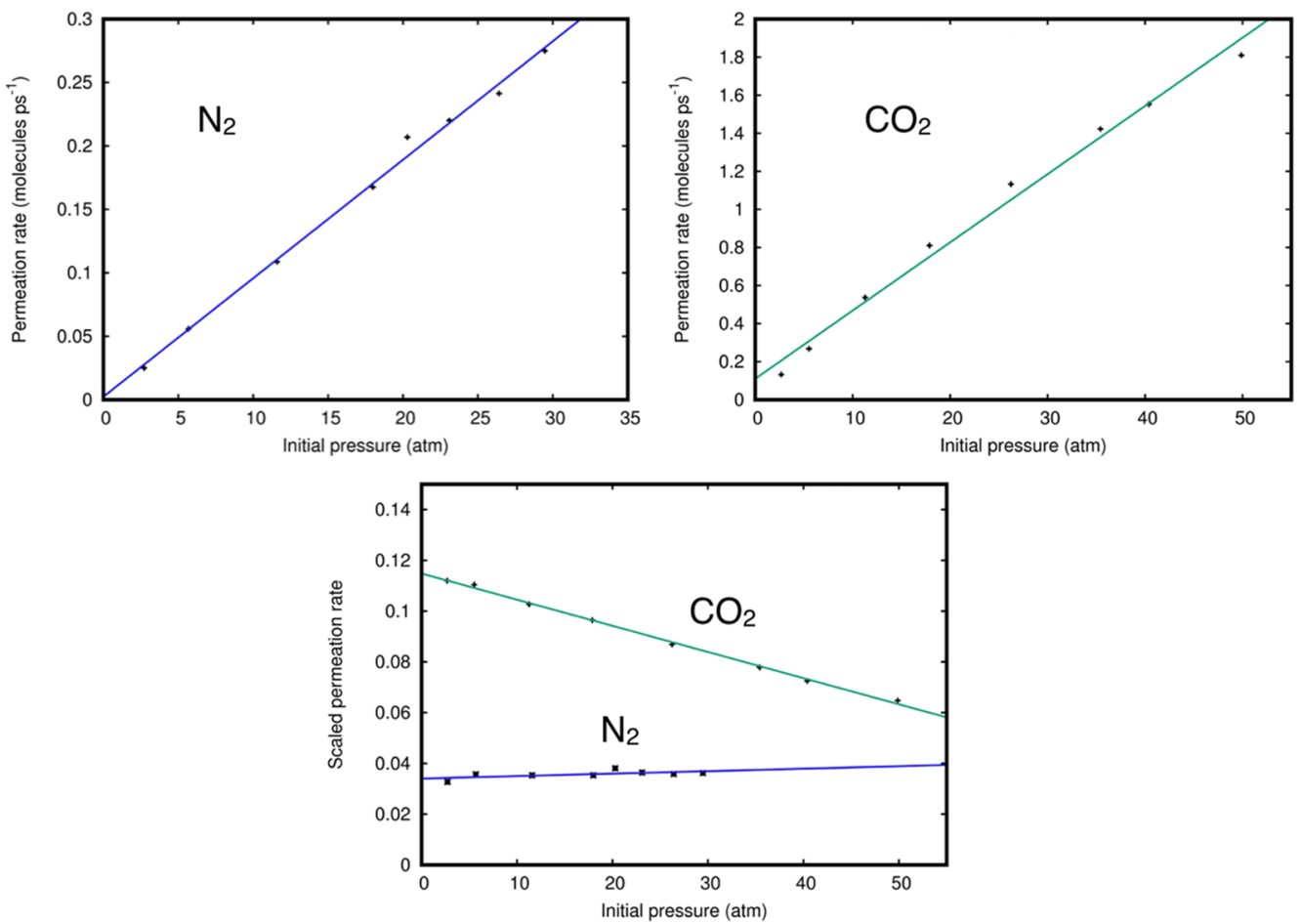

Figure 7. Permeation rate through the single-layer membrane as a function of initial pressure for pure $\mathrm{N}_{2}$ (left panel) and pure $\mathrm{CO}_{2}$ (right panel) gas systems. The lower panel shows the permeation rates scaled by the gas uptake (see Supporting Information, Table S5).

To widen the range of the experimental conditions, we performed simulations using eight different values of the initial pressure, varying the number of gas molecules in the box, see Tables S3 and S4 in the Supporting Information, to examine the dependence of the permeation rate on pressure. The results are shown in Figure 7, upper panels, where it can be seen that the permeation rate varies linearly with the initial pressure for both pure $\mathrm{CO}_{2}$ and pure $\mathrm{N}_{2}$ systems. The lower panel of Figure 7 shows the permeation rate scaled by the average gas uptake at the same pressure (Table S5 in the Supporting Information) reported as a function pressure for both gases. This scaled rates can be thought of as related to the crossing probability of an adsorbed molecule. For both molecules, the probability does not increase (as could be expected), indicating that a saturation effect occurs: when the number of absorbed molecules increases significantly, the larger fraction of occupied adsorption sites may hinder to some extent the crossing occurring from the two sides of the membrane. The effect is more pronounced for $\mathrm{CO}_{2}$ (for which the trend is decreasing) because of higher average uptake numbers. Indeed, $\mathrm{CO}_{2}$ molecules in a physisorption state have longer lifetimes (wait more time before crossing) because of the combined effect of the stronger attraction and the strict stereoselective character of the crossing dynamics, a consequence of the high-energy barriers that the molecule encounters approaching the center of the pore with orientations other than the perpendicular one (see Figures 1 and 2 and section 2). The adsorption is lessefficient, and the stereoselective requirements are much looser in the case of $\mathrm{N}_{2}$ for which this effect is less pronounced.

We can estimate the value of the permeance from the slope obtained by linear regressions of the data shown in Figure 7, where the linear-fitting functions are also reported. The obtained permeance values, given in GPU units ( 1 gas permeance unit $=3.35 \times 10^{-10} \mathrm{~mol} \mathrm{~m}^{-2} \mathrm{~s}^{-1} \mathrm{~Pa}^{-1}$ ), are 8.60 $\times 10^{7}$ and $2.02 \times 10^{7} \mathrm{GPU}$, for $\mathrm{CO}_{2}$ and $\mathrm{N}_{2}$, respectively. The so-called permeation ratio $\left(\mathrm{CO}_{2} / \mathrm{N}_{2}\right)$ for the two gases is 4.25 , meaning that the number of permeations of $\mathrm{CO}_{2}$ is typically 4.25 times that of $\mathrm{N}_{2}$. This propensity is reflected in the $z$ density profiles in Figure 6 as well as in the adsorption isotherms reported in Figure 8.

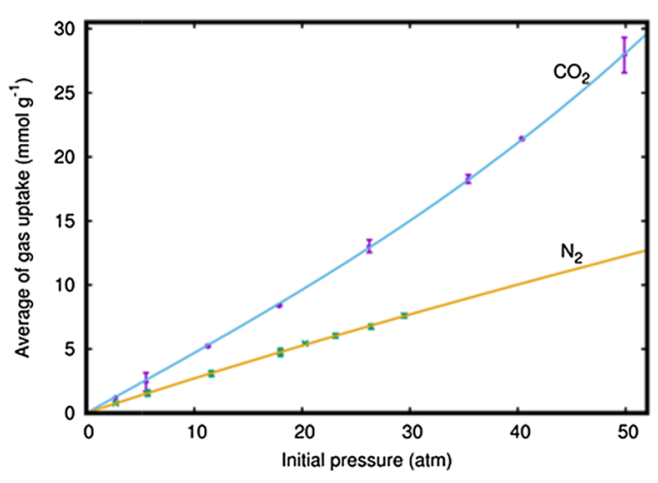

Figure 8. Adsorption isotherms of the single-layer membrane for pure $\mathrm{N}_{2}$ and $\mathrm{CO}_{2}$ systems, as a function of pressure.

The average number of gas uptakes in Figure 8 was calculated by integrating the $z$-density profile considering absorbed any gas molecule located in the range $\pm 6.9 \AA$ with respect to the membrane position. This distance range, where the physisorption precursor state can be effectively formed (later it will be defined as adsorption region), is assumed to be equal to 2 times the interlayer equilibrium distance of the graphtriyne bilayer. The complete data set regarding the gas permeance and uptake values for the monolayer can be found in the Supporting Information, Tables S5-S14. The observed $\mathrm{CO}_{2} / \mathrm{N}_{2}$ permeance ratio (about 4.25) is lower than the $\mathrm{CO}_{2} /$ $\mathrm{N}_{2}$ permeance ratio reported by Liu et al. ${ }^{14}$ for nanoporous graphene, where the estimated ratio is 100 . However, 

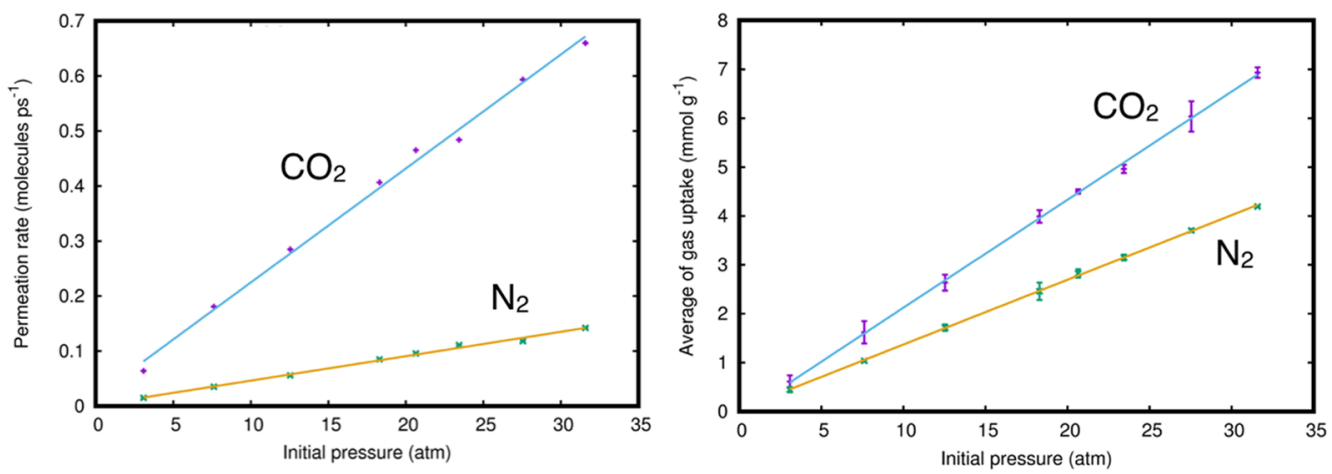

Figure 9. Permeation rate (left panel) and adsorption isotherm (right panel) for the single-layer membrane with the $\mathrm{CO}_{2} / \mathrm{N}_{2}$ gas mixture system as a function of the initial pressure.

graphtriyne exhibits for $\mathrm{CO}_{2}$ a permeance 2 orders of magnitudes higher than that of the corresponding nanoporous graphene $\left(2.8 \times 10^{5} \mathrm{GPU}\right)$. This behavior obviously depends on the pore size of the membrane and on the position and strength of the repulsive component associated to the intermolecular interactions.

To obtain the permeation selectivity of $\mathrm{CO}_{2} / \mathrm{N}_{2}$ for the single-layer system, we performed simulations of mixtures at different pressures and fixed composition of $50 \% \mathrm{CO}_{2}$ and $50 \%$ $\mathrm{N}_{2}$. Figure 9 (left panel) shows that the permeation rate varies linearly with the initial pressure of the mixture. From the slope, the permeance values for $\mathrm{CO}_{2}$ and $\mathrm{N}_{2}$ result to be $4.49 \times 10^{7}$ and $0.96 \times 10^{7} \mathrm{GPU}$, respectively, whereas the $\mathrm{CO}_{2} / \mathrm{N}_{2}$ permeation selectivity ratio is 4.67 . It can be seen that the permeance values for the mixture are always lower because of the competition between $\mathrm{CO}_{2}$ and $\mathrm{N}_{2}$ interacting with the membrane.

This means that $\mathrm{CO}_{2}-\mathrm{N}_{2}$ mutual interactions delay the crossing processes, setting the conditions for $\mathrm{CO}_{2}$ to stay preferentially near the surface as a consequence of the major stability of its physisorption states, as it can be seen from the $z$ density profile of the mixture depicted in Figure 10 and in the

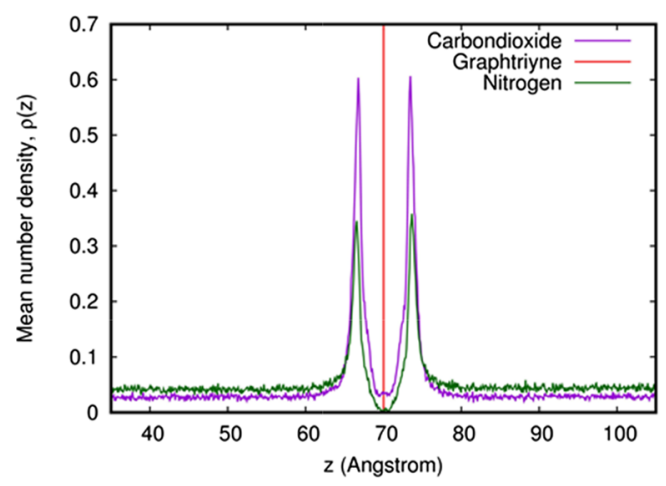

Figure 10. Comparison of the $z$-density profiles at 18.297 atm for the single-layer membrane with the $\mathrm{CO}_{2} / \mathrm{N}_{2}$ gas mixture system.

adsorption isotherm of Figure 9 (right panel). As a result, $\mathrm{CO}_{2}$ undergoes a higher number of permeation events than $\mathrm{N}_{2}$, and the $\mathrm{CO}_{2} / \mathrm{N}_{2}$ permeation selectivity is slightly higher than the $\mathrm{CO}_{2} / \mathrm{N}_{2}$ ratio as obtained from the permeance values of pure systems. The adsorption coefficients can be calculated from the slope of the adsorption isotherms, for both pure and mixed systems, resulting to be for $\mathrm{CO}_{2} 0.56$ and $0.22 \mathrm{mmol} \mathrm{g}^{-1}$ $\mathrm{atm}^{-1}$, respectively, and for $\mathrm{N}_{2} 0.25$ and $0.13 \mathrm{mmol} \mathrm{g}^{-1} \mathrm{~atm}^{-1}$, respectively. The difference in the adsorption coefficient values for pure and mixed system is, again, because of the $\mathrm{CO}_{2}-\mathrm{N}_{2}$ interaction effect.

Multilayer Membranes. Unlike the single-layer membranes, those composed of bi- and trilayers have an additional feature that is an extra space between the layers. Therefore, it can be expected that this interlayer space could work as an adsorbing region to host gas molecules. In Figure 11, the $z$ density plots of pure gases with bilayer (upper panels) and trilayer (lower panels) membranes are reported. The previous hypothesis is clearly supported by the results for the $\mathrm{CO}_{2}$ molecules because the density in the interlayer region achieves significant peaks and the area under the curve is large, especially for the triple-layer systems (see lower panels in Figure 11). The plots also indicate that the interlayer region adsorbs $\mathrm{CO}_{2}$ much more efficiently than $\mathrm{N}_{2}$. Consistent with this picture are the adsorption isotherms related to the interlayer region for the pure gas systems, shown in Figure 12. In the case of $\mathrm{CO}_{2}$, the adsorption isotherms are not linear, whereas a linear trend is still observed for $\mathrm{N}_{2}$. Indeed, the strong adsorption, favored also by the high packing degree (consider the much wider potential well for $\mathrm{CO}_{2}$ in Figures 1 and 2), makes $\mathrm{CO}_{2}$ to saturate the pores for increasing the initial pressure. The adsorbed molecules hinder the crossing process when almost all of the interlayer regions are occupied. This saturation effect is not observed in the case of $\mathrm{N}_{2}$, for which the adsorption is less-efficient.

Permeation rates of $\mathrm{N}_{2}$ shown for bilayer and trilayer membranes in Figure 12 are significantly higher than those obtained for the monolayer (Figure 7), because, due to the interlayer adsorption, molecules are stabilized near the membrane and the probability of crossing for $\mathrm{N}_{2}$ increases because of low-penetration barriers (see Figures 1 and 2).

Although in the case of pure $\mathrm{CO}_{2}$, the adsorption isotherms are not linear, the gas permeance can be still estimated by linear regression in the low-pressure range, before the onset of saturation. The permeance values for pure gases with bi- and trilayer membranes are listed in Table 1 and compared with those obtained for the monolayer membrane.

The reported data indicate that for pure $\mathrm{N}_{2}$, the permeance increases as the number of layers increases. This means that, adding layers, the attraction for $\mathrm{N}_{2}$ becomes stronger, which is consistent with the interaction energies reported in ref 26 (about 112.5 and $205.0 \mathrm{meV}$ for single and triple layer, respectively). A partly similar behavior is observed for $\mathrm{CO}_{2}$ because the permeance increases in passing from the single layer to bilayer as a result of the increasing attraction but 

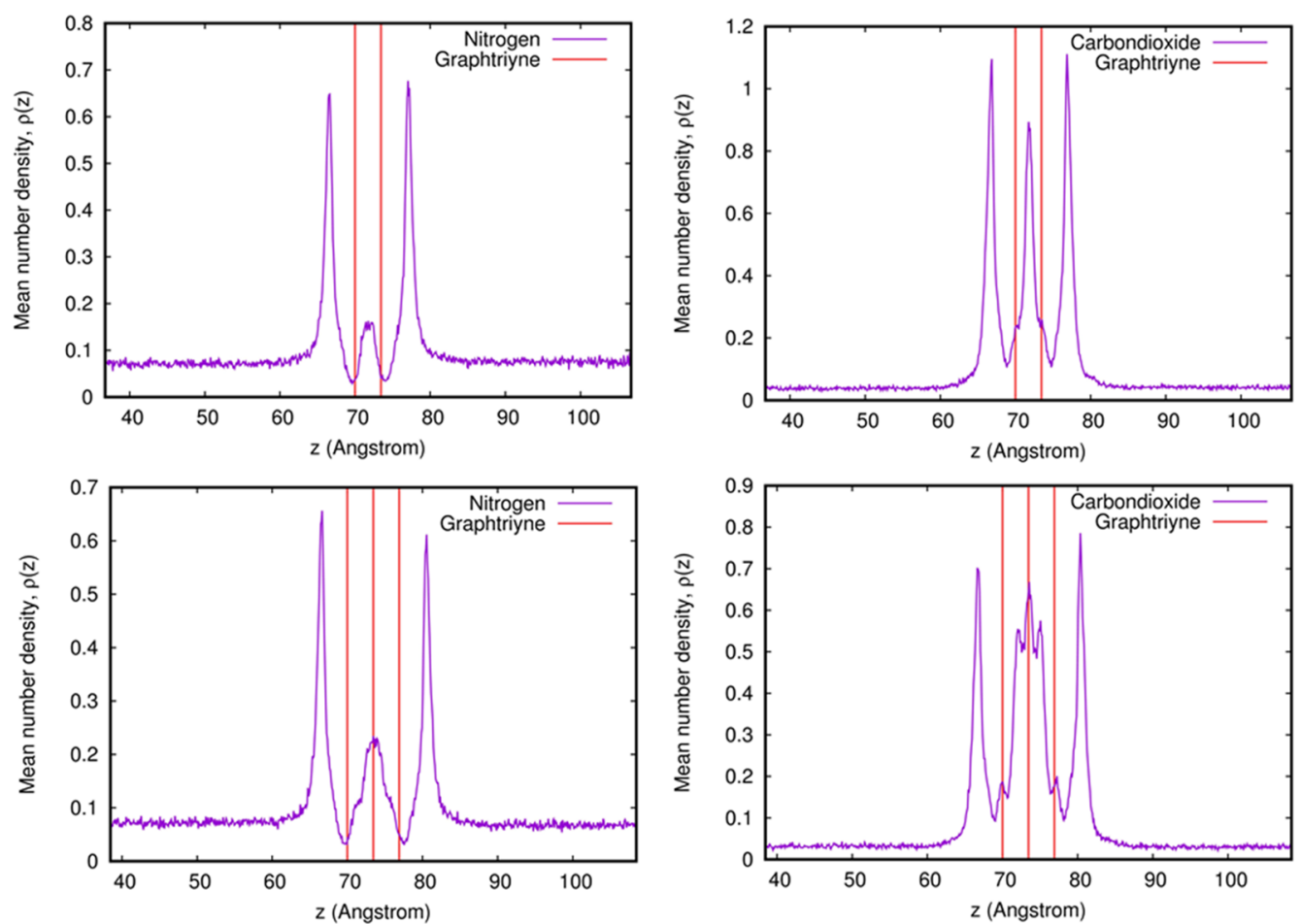

Figure 11. $z$-Density profiles for pure $\mathrm{N}_{2}$ at $17.99 \mathrm{~atm}$ (left panels) and for pure $\mathrm{CO}_{2}$ at $17.88 \mathrm{~atm}$ (right panels) obtained considering both bilayer (upper panels) and trilayer (lower panels) membranes.
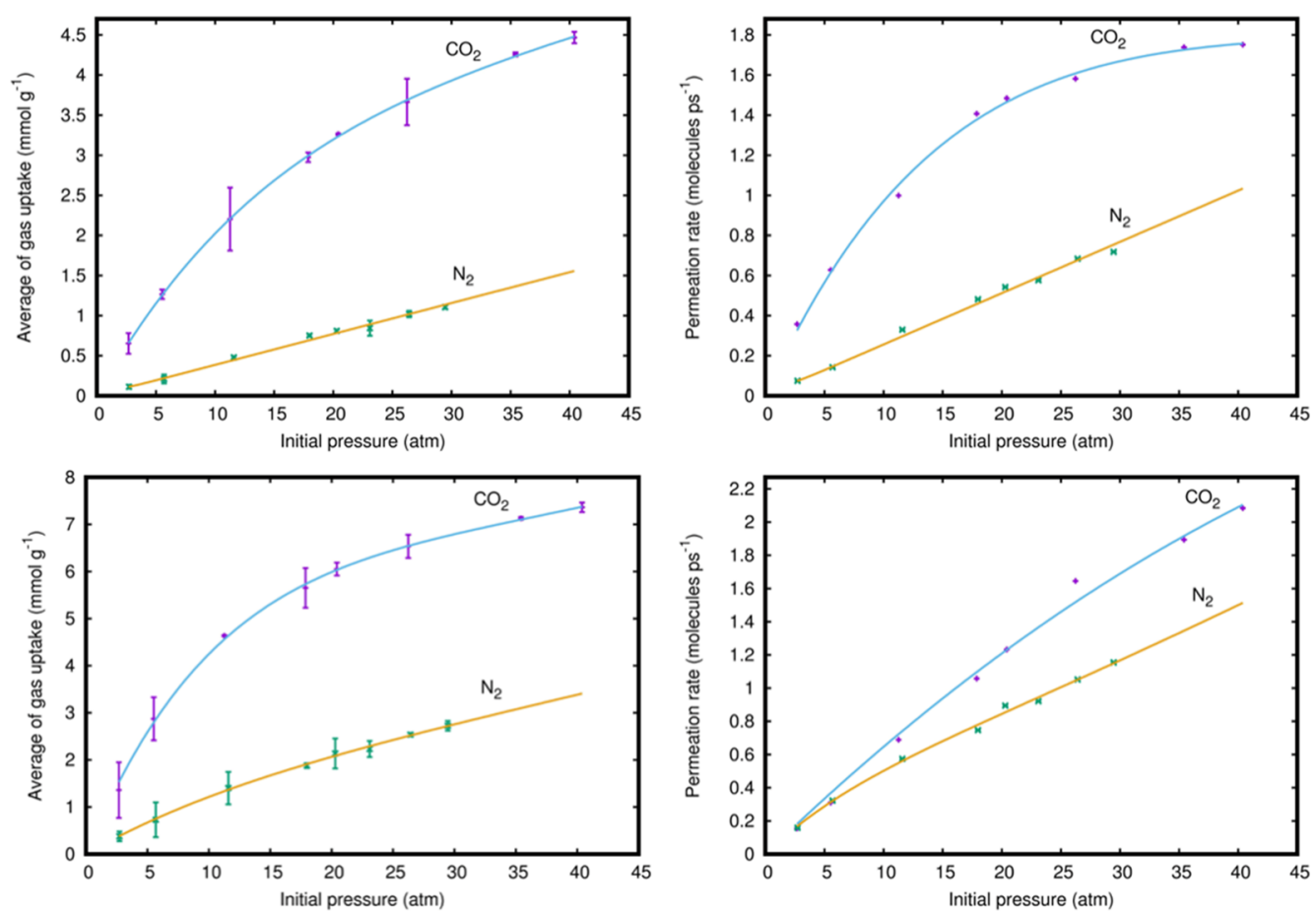

Figure 12. Adsorption isotherms related to the interlayer region (left panels) and permeation rate as a function of the initial pressure (right panels) for pure $\mathrm{CO}_{2}$ and $\mathrm{N}_{2}$ considering both bilayer (upper panels) and trilayer (lower panels) membranes.

decreases passing from the bilayer to the trilayer membrane. This decrease in the $\mathrm{CO}_{2}$ permeance depends on the adsorption in the interlayer region. Therefore, besides calculating the adsorption isotherm in the interlayer region (Figure 12), we also estimated a total adsorption isotherm, obtained by adding the adsorption occurring outside the membrane, controlled by the stability of the physisorption states, to those occurring in the interlayer region, conditioned to the permeation process. The total adsorption isotherm for both $\mathrm{N}_{2}$ and $\mathrm{CO}_{2}$ varies linearly with the initial pressure; thus, using linear regression, we could obtain the total adsorption coefficients. The total adsorption coefficients of $\mathrm{CO}_{2}$ for bilayer and trilayer membranes, equal to 0.64 and $0.69 \mathrm{mmol}$ $\mathrm{g}^{-1}$ atm $^{-1}$, respectively, are higher than those of $\mathrm{N}_{2}, 0.32$ and 
Table 1. Gas Permeance for Pure Gas Systems (in Units of $10^{7}$ GPU)

\begin{tabular}{lrc}
\multicolumn{1}{c}{ system } & gas permeance $\left(\times 10^{7} \mathrm{GPU}\right)$ & $\mathrm{CO}_{2} / \mathrm{N}_{2}$ ratio \\
\hline single layer, $\mathrm{CO}_{2}$ & $8.6 \pm 0.3131$ & $4.2 \pm 0.2836$ \\
single layer, $\mathrm{N}_{2}$ & $2.02 \pm 0.0614$ & \\
bilayer, $\mathrm{CO}_{2}$ & $14.7 \pm 0.8126$ & $2.7 \pm 0.2490$ \\
bilayer, $\mathrm{N}_{2}$ & $5.3 \pm 0.1891$ & \\
trilayer, $\mathrm{CO}_{2}$ & $13.6 \pm 0.2816$ & $1.7 \pm 0.1063$ \\
trilayer, $\mathrm{N}_{2}$ & $7.8 \pm 0.3190$ & \\
\hline
\end{tabular}

$0.36 \mathrm{mmol} \mathrm{g}^{-1} \mathrm{~atm}^{-1}$, respectively. These values are consistent with the decrease in the value of the $\mathrm{CO}_{2}$ permeance observed in passing from the bilayer to the trilayer membrane.

Similar results were also observed for the $\mathrm{CO}_{2} / \mathrm{N}_{2}$ mixture system. The interlayer region provides a stronger adsorption of $\mathrm{CO}_{2}$ with respect to $\mathrm{N}_{2}$, as shown in the $z$-density plots of Figure 13 and in the adsorption isotherms given in Figure 14. The adsorption isotherms, related to the interlayer region (left panels in Figure 14), also show that the $\mathrm{CO}_{2}$ uptake of the trilayer membrane (bottom left panel) is approximately twice the bilayer one (see upper left panel). This is consistent with the fact that in the trilayer membrane, the volume between outer layers is twice that of the bilayer one (observe it qualitatively from the simulation snapshots represented in Figure 13). From the total adsorption isotherms, also given in Figure 14 (right panels), we obtained the adsorption coefficients of $\mathrm{CO}_{2}$ for the bilayer and trilayer membranes equal to 0.29 and $0.32 \mathrm{mmol} \mathrm{g}^{-1} \mathrm{~atm}^{-1}$, respectively, and for $\mathrm{N}_{2}$ corresponding values of 0.16 and $0.17 \mathrm{mmol} \mathrm{g}^{-1} \mathrm{~atm}^{-1}$.

Table 2 summarizes the adsorption coefficients obtained from the total adsorption isotherms for pure gases and mixtures. The adsorption coefficients of the mixtures are lower than those of the pure systems. The $\mathrm{CO}_{2}-\mathrm{N}_{2}$ competition to interact with the membrane explains this drop. The permeance values for the gaseous mixtures are shown in Table 3, where it can be seen that the values for both $\mathrm{N}_{2}$ and $\mathrm{CO}_{2}$ show the same trend as in pure systems (see Table 1). Again, those phenomena can be correlated with the combination of molecular crossing and to capture the events.

Table 3 also lists the $\mathrm{CO}_{2} / \mathrm{N}_{2}$ mixture permeance selectivity. The permeance selectivity increases in passing from single to bilayer membranes, and then decreases for the trilayer ones. To further characterize the behavior of the membranes, it is expedient to consider the adsorption selectivity $S_{\mathrm{ads}}{ }^{\mathrm{CO}_{2} / \mathrm{N}_{2}}$ calculated using the following formula ${ }^{10}$

$$
S_{\mathrm{ads}}^{\mathrm{CO}_{2} / \mathrm{N}_{2}}=\frac{n_{\mathrm{CO}_{2}(\text { ads })}}{n_{\mathrm{CO}_{2}(\text { free })}} \times \frac{n_{\mathrm{N}_{2}(\text { free })}}{n_{\mathrm{N}_{2}(\text { ads })}}
$$

where $n_{\mathrm{CO}_{2} \text { (ads) }}$ and $n_{\mathrm{N}_{2} \text { (ads) }}$ represent the average numbers of adsorbed $\mathrm{CO}_{2}$ and $\mathrm{N}_{2}$ molecules, respectively, and $n_{\mathrm{CO}_{2} \text { (free) }}$ and $n_{\mathrm{N}_{2} \text { (free) }}$ represent the average numbers of free $\mathrm{CO}_{2}$ and $\mathrm{N}_{2}$ molecules, respectively. The numbers of adsorbed gas molecules can be obtained by integrating the $z$-density functions over the adsorbing regions, whereas the numbers of free gas molecules can be calculated from the difference between the total number of molecules and the number of adsorbed ones. By averaging both such values over the two membranes contained in the simulation box, we obtain the corresponding mean values to be used in eq 1 . Figure 15 shows the plots as a function of the pressure of the interlayer adsorption selectivity, for which the adsorbing regions are located between the layers, and the total adsorption selectivity, for which the adsorbing regions are obtained considering the interlayer regions plus the range $\pm 6.9 \AA$ (twice the interlayer equilibrium distance of the graphtriyne bilayer stacking) from
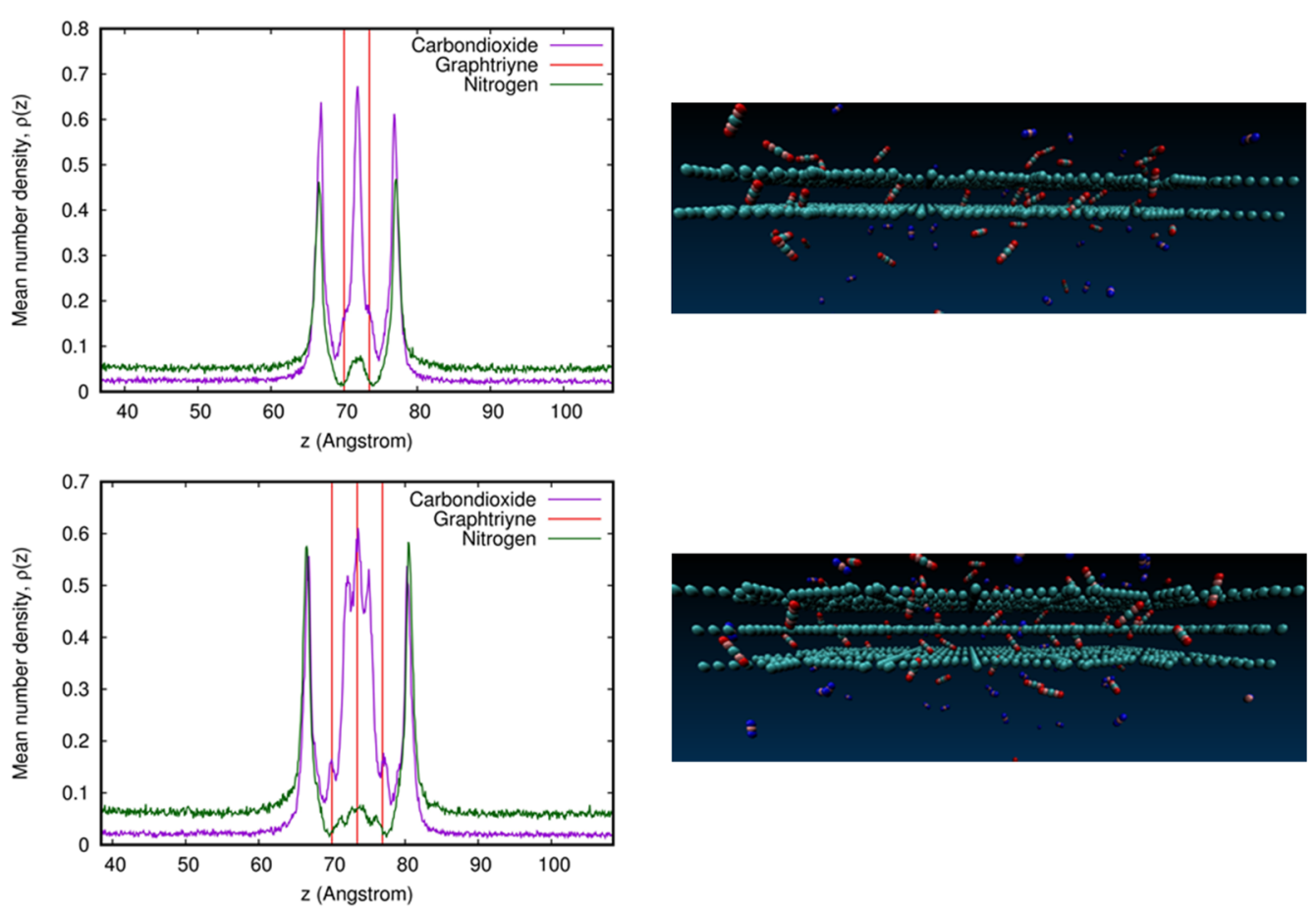

Figure 13. Snapshots of configurations (right) and $z$-density profiles (left) for the $\mathrm{CO}_{2} / \mathrm{N}_{2}$ mixture with bilayer (upper panel) and trilayer (lower panel) membranes, calculated at 23.437 and $27.548 \mathrm{~atm}$, respectively. In the snapshots, the $z$-axis is perpendicular to the horizontal plane, where the membranes are laying. 

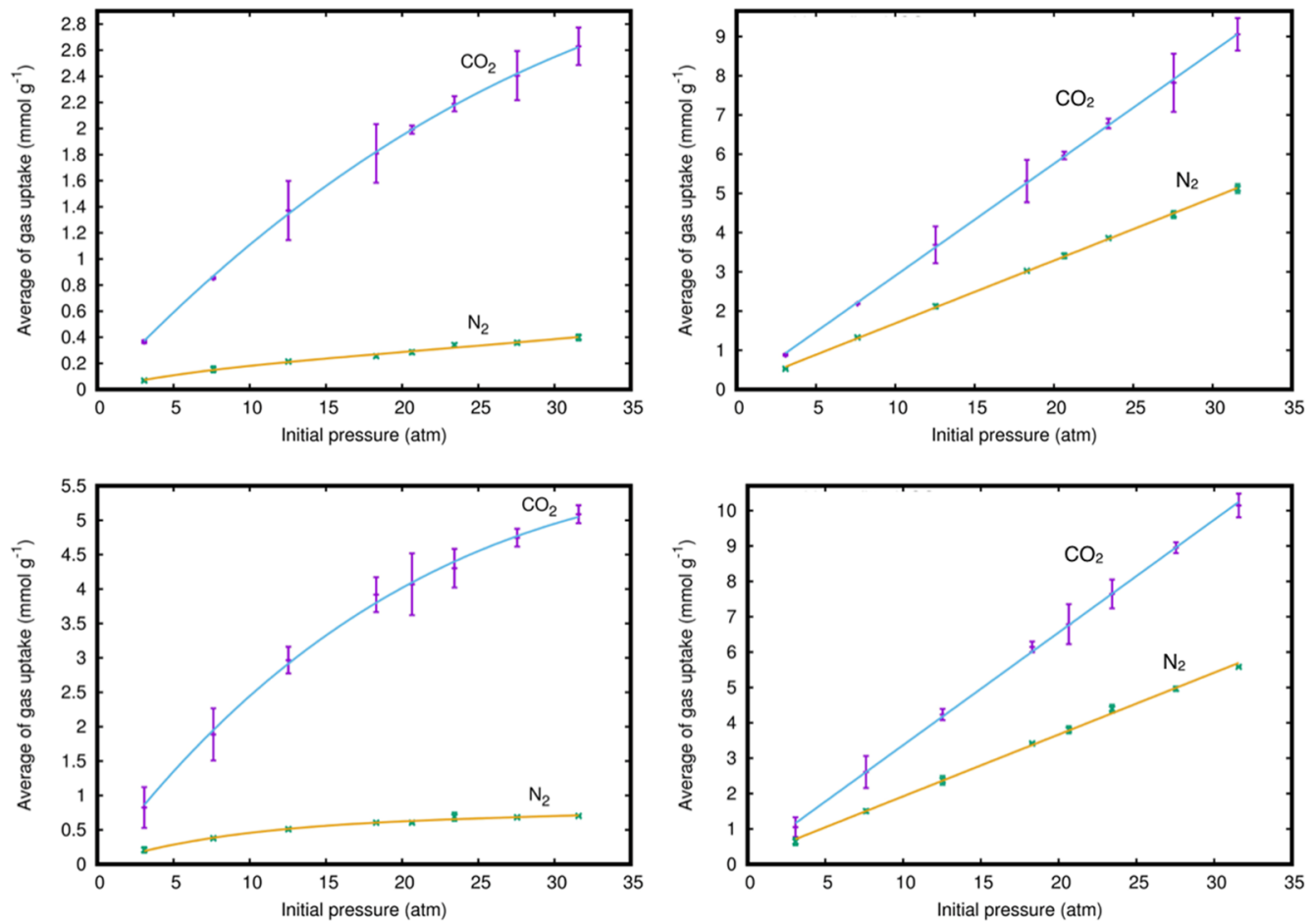

Figure 14. Interlayer region adsorption isotherms (left panels) and total adsorption isotherms (right panels) for the $\mathrm{CO}_{2} / \mathrm{N}_{2}$ mixture system. Upper panels refer to the bilayer membrane and lower panels to the trilayer one.

Table 2. Adsorption Coefficients $\left(\mathrm{mmol} \mathrm{g}^{-1} \mathrm{~atm}^{-1}\right)$ of $\mathrm{CO}_{2}$ and $\mathrm{N}_{2}$ in the Pure and Mixture Systems

\begin{tabular}{lcc}
\multicolumn{1}{c}{ system } & pure systems & mixture systems \\
single layer, $\mathrm{CO}_{2}$ & $0.56 \pm 0.0149$ & $0.221 \pm 0.0025$ \\
single layer, $\mathrm{N}_{2}$ & $0.256 \pm 0.0031$ & $0.132 \pm 0.0010$ \\
bilayer, $\mathrm{CO}_{2}$ & $0.644 \pm 0.0087$ & $0.285 \pm 0.0022$ \\
bilayer, $\mathrm{N}_{2}$ & $0.316 \pm 0.0036$ & $0.160 \pm 0.0013$ \\
trilayer, $\mathrm{CO}_{2}$ & $0.693 \pm 0.0084$ & $0.318 \pm 0.0033$ \\
trilayer, $\mathrm{N}_{2}$ & $0.360 \pm 0.0049$ & $0.174 \pm 0.0030$ \\
\hline
\end{tabular}

Table 3. Gas Permeance for Mixture Systems

\begin{tabular}{cccc} 
& \multicolumn{2}{c}{ gas permeance (units of $\left.10^{7} \mathrm{GPU}\right)$} & \\
\cline { 2 - 3 } system & $\mathrm{CO}_{2}$ & $\mathrm{~N}_{2}$ & $\begin{array}{c}\text { permeance } \\
\text { selectivity }\end{array}$ \\
$\begin{array}{c}\text { single layer, } \\
\mathrm{CO}_{2} / \mathrm{N}_{2}\end{array}$ & $4.5 \pm 0.1285$ & $0.96 \pm 0.0296$ & $4.7 \pm 0.2775$ \\
bilayer, $\mathrm{CO}_{2} / \mathrm{N}_{2}$ & $10.3 \pm 0.6224$ & $1.9 \pm 0.1084$ & $5.4 \pm 0.6264$ \\
trilayer, $\mathrm{CO}_{2} / \mathrm{N}_{2}$ & $7.5 \pm 0.3596$ & $2.8 \pm 0.2721$ & $2.7 \pm 0.3793$ \\
\hline
\end{tabular}

the outermost layers. The total adsorption selectivity of the monolayer membrane is also reported for comparison.

We can see that total adsorption selectivity increases with the number of layers. For single and bilayer membranes, the selectivity does not change significantly, when the initial pressure increases (see right panel in Figure 15). On average, the single-layer membrane provides a selectivity equal to about 2 , meaning the ratio between the average numbers of adsorbed and free molecules for $\mathrm{CO}_{2}$ is 2 times higher than for $\mathrm{N}_{2}$. The selectivity of the bilayer membrane is about 4 . The trend is different for the trilayer membrane, showing at low pressures a high selectivity of about 8 that drops to 5 when the pressure increases.

Overall, the results about selectivity indicate that, at low pressures, graphtriyne trilayer membranes perform an effective discrimination of $\mathrm{CO}_{2}$ through adsorption, whereas the bilayer ones can act as a good molecular sieve, as the data in Table 3 confirm.

The graphtriyne bilayer membrane can be compared with fluorine-modified porous graphenes, which have been investigated in ref 21 . Under similar conditions, the partially modified pore-22 (pore obtained removing 22 carbon atoms and half of the dangling bonds saturated with fluorine atoms) reaches a permeance selectivity of about 4, whereas the completely modified pore-22 (all bonds saturated with fluorine atoms) has a selectivity about 11 . Although the permeance selectivity of the graphtriyne bilayer membrane (approximately 5.36, see Table 4) is lower than 11 , it has a much higher $\mathrm{CO}_{2}$ permeance $\left(10.31 \times 10^{7} \mathrm{GPU}\right)$ than the completely passivated pore-22 $\left(\sim 7 \times 10^{6} \mathrm{GPU}\right)$. Therefore, it should be more efficient to use it as an initial membrane for $\mathrm{CO}_{2}$ postcombustion separation. The trilayer membrane, although less efficient as a molecular sieve (see Table 3), is suitable to be used as an adsorption medium to capture $\mathrm{CO}_{2}$ in postcombustion mixtures. This idea is supported by the fact that the trilayer membrane exhibits both relatively high interlayer adsorption and total uptake selectivity (Figure 15) and also a relatively high $\mathrm{CO}_{2}$ uptake (Figure 13).

A comparison of the performance of graphtriyne multilayer membranes with other materials, for $\mathrm{CO}_{2}$ separation and capture, is provided in Table 4. Using MD simulations, we estimated the total gaseous uptake to be approximately 17.69 $\mathrm{mmol} \mathrm{g}^{-1}$ at $26.59 \mathrm{bar}$ and $300 \mathrm{~K}$ for graphtriyne trilayer membranes, in the presence of pure $\mathrm{CO}_{2}$. The total $\mathrm{CO}_{2}$ uptake values of the trilayer membrane (available in the Supporting Information, Tables S9 and S10) are compared with those for porous carbons reported in refs, ${ }^{12,50,51}$ evaluated under similar conditions. Moreover, the total $\mathrm{CO}_{2}$ uptake values of the trilayer membrane are also comparable or even higher than those of some covalent organic polymers (COPs) 

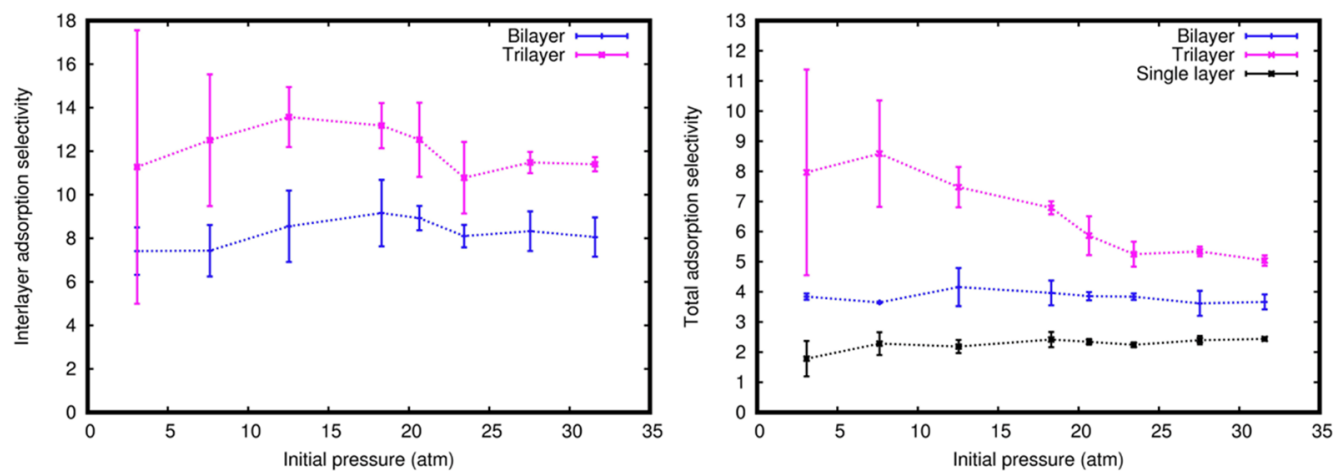

Figure 15. Interlayer adsorption selectivity (left panel) and total adsorption selectivity (right panel) of the mixture system plotted as a function of initial pressure.

Table 4. Performance Comparison of Multilayer Graphtriyne and Some Carbon-Based Materials from Different Theoretical Studies $^{a}$

\begin{tabular}{|c|c|c|}
\hline materials & $\mathrm{CO}_{2} / \mathrm{N}_{2}$ selectivity & $\mathrm{CO}_{2}$ uptake $\left(\mathrm{mmol} \mathrm{g}^{-1}\right)$ \\
\hline \multicolumn{3}{|c|}{ Permeance } \\
\hline graphtriyne bilayers\# & 5.4 & \\
\hline F-modified porous graphene ${ }^{21}$ & $4.0-11.0$ & \\
\hline \multicolumn{3}{|c|}{ Adsorption } \\
\hline graphtriyne trilayers\# & $11.3-12.5^{*}(3.12-20.92$ bar; $300 \mathrm{~K})$ & $11.82-17.69^{* *}(18.12-26.59 \mathrm{bar} ; 300 \mathrm{~K})$ \\
\hline functionalized graphitic slit pores ${ }^{10}$ & $5.0-20.0(20$ bar; $298 \mathrm{~K})$ & \\
\hline $\mathrm{COPs}^{7}$ & $8.4-13.7(1.01$ bar; $313 \mathrm{~K})$ & $4.61-17.43(18$ bar; $298 \mathrm{~K})$ \\
\hline MOFs $^{11,51}$ & $5.0-40.0(20$ bar; $298 \mathrm{~K})$ & $10.00-30.00(25$ bar; $298-302 \mathrm{~K})$ \\
\hline \multirow[t]{2}{*}{ porous carbons ${ }^{49,50}$} & & $16.10-21.10(20$ bar; $298 \mathrm{~K})$ \\
\hline & & $7.51-18.80(20$ bar; $297 \mathrm{~K})$ \\
\hline hierarchical porous carbons (HPCs) ${ }^{12}$ & & $10.00-21.00(20$ bar; $300 \mathrm{~K})$ \\
\hline
\end{tabular}

and MOFs presented in refs 7 and 11 , respectively. For $\mathrm{CO}_{2} /$ $\mathrm{N}_{2}$ gas mixtures, our estimation of the interlayer adsorption selectivity (about 12.5 at 20.92 bar, and 11.3 at 3.12 bar, see Figure 15 , both at $300 \mathrm{~K}$ ) is higher than that of graphitic slit pores (around 5 at 20 bar and $298 \mathrm{~K}$ ). From the data in Table 4 , it emerges that the adsorption selectivity of multilayer graphtriyne membranes is also comparable with that of some functionalized graphitic slit pores reported also by Liu et al. ${ }^{10}$ (approximately 20 at 20 bar and $298 \mathrm{~K}$ ), of some COPs (in range of $8.4-13.7$ at $1.01 \mathrm{bar}$ and $313 \mathrm{~K}$ ), and of some MOFs. ${ }^{52}$ Considering the properties of carbon-based materials (hydrophobic, inert, and thermally stable), graphtriyne multilayers can be considered as a promising alternative for postcombustion $\mathrm{CO}_{2}$ separation and capture materials.

All of the averaged quantities obtained from simulations (not reported in the paper) are made available in the Supporting Information (Tables S3-S14).

\section{CONCLUSIONS}

The performance of graphtriyne-based membranes for $\mathrm{CO}_{2} /$ $\mathrm{N}_{2}$ separation has been investigated by extensive MD simulations based on a new formulation of the force fields required to describe the interactions in gas phase and between the molecules and the layers. The resulting PES provides an accurate description of the interactions and permits realistic simulations of the physisorption states of molecules and of the molecular attraction and repulsion between the pore. The model is a quantitative tool for the estimation of membrane properties, such as gas uptake, permeance, and selectivity in view of applications for postcombustion mixture separation and can be easily extended to carbon nanostructures interacting with a variety of different gaseous mixtures. We found that graphtriyne bilayer membranes have a high permeance (on the order of magnitude of $10^{7} \mathrm{GPU}$ ) with a relatively good selectivity (about 5.36). Adsorbed $\mathrm{CO}_{2}$ lowers the permeance of the membrane when the pressure is high. Therefore, at low pressure, graphtriyne bilayer membranes exhibit good performance as an initial molecular sieve candidate for postcombustion $\mathrm{CO}_{2}$ separation.

As the number of layers increases, the membrane strongly attracts the gas molecules, especially in the interlayer region. Our simulations have shown that graphtriyne trilayer membranes have a relatively high interlayer adsorption selectivity (11.3-13.4 in the range of 3.08-12.54 atm) and high $\mathrm{CO}_{2}$ uptake. The average of $\mathrm{CO}_{2}$ uptake linearly grows with the initial pressure, which gives related adsorption coefficients of around 0.69 and $0.32 \mathrm{mmol} \mathrm{g}^{-1} \mathrm{~atm}^{-1}$ for pure and mixture systems, respectively. These results suggest that graphtriyne $n$-layer $(n \geq 3)$ membranes are comparable and competitive with other carbon-based adsorbing materials for postcombustion $\mathrm{CO}_{2}$ capture.

\section{ASSOCIATED CONTENT}

\section{Supporting Information}

The Supporting Information is available free of charge on the ACS Publications website at DOI: 10.1021/acs.jpcc.8b04960.

Parameters of the used force field; comparison with the standard AMBER force field; complete data set obtained from simulations of 1-2-3-layer systems with pure gases 
and mixtures, including permeance and uptake numbers (PDF)

\section{AUTHOR INFORMATION}

\section{Corresponding Author}

*E-mail: Noelia.faginaslago@unipg.it.

\section{ORCID $\odot$}

Yusuf Bramastya Apriliyanto: 0000-0003-0683-8456

Noelia Faginas Lago: 0000-0002-4056-3364

Stefano Evangelisti: 0000-0001-8782-443X

Thierry Leininger: 0000-0002-7373-0966

\section{Notes}

The authors declare no competing financial interest.

\section{ACKNOWLEDGMENTS}

Y.B.A. gratefully acknowledges the financial support from the "Theoretical Chemistry and Computational Modelling" (TCCM) Erasmus-Plus Master program. N.F.L. acknowledges the financial support from Fondazione Cassa di Risparmio di Perugia (P 2014/1255, ACT 2014/6167). S.E. and T.L. acknowledge the "Programme Investissements d'Avenir" ANR11-IDEX-0002-02, reference ANR-10-LABX-0037-NEXT for financial support. A.L. acknowledges support for "Fondazione Cassa Risparmio Perugia (Codice Progetto: 2015.0331.021 Ricerca Scientifica e Tecnologica)", from Fondo Ricerca di Base 2014 of the Università di Perugia and from MIUR PRIN 2015 (contract 2015F59J3R 002). A.L. and N.F.L. also thank the OU Supercomputing Center for Education \& Research (OSCER) at the University of Oklahoma, for allocated computing time. N.F.L., A.L., and F.P. thank MIUR and the University of Perugia for the financial support of the AMIS project through the program "Dipartimenti di Eccellenza". M.B. acknowledges support from the FIS2017-84391-C2-2-P Spanish Grant. Allocation of computing time by CESGA (Spain) is also acknowledged.

\section{REFERENCES}

(1) Ganin, A. Y.; Takabayashi, Y.; Jeglič, P.; Arčon, D.; Potočnik, A.; Baker, P. J.; Ohishi, Y.; McDonald, M. T.; Tzirakis, M. D.; McLennan, A.; Darling, G. R.; Takata, M.; Rosseinsky, M. J.; Prassides, K. Polymorphism control of superconductivity and magnetism in Cs3C60 close to the Mott transition. Nature 2010, 466, 221-225.

(2) Nakamura, A.; Ohtsuka, J.; Miyazono, K.-i.; Yamamura, A.; Kubota, K.; Hirose, R.; Hirota, N.; Ataka, M.; Sawano, Y.; Tanokura, M. Improvement in quality of protein crystals grown in a high magnetic field gradient. Cryst. Growth Des. 2012, 12, 1141-1150.

(3) Potticary, J.; Terry, L. R.; Bell, C.; Papanikolopoulos, A. N.; Christianen, P. C. M.; Engelkamp, H.; Collins, A. M.; Fontanesi, C.; Kociok-Köhn, G.; Crampin, S.; et al. An unforeseen polymorph of coronene by the application of magnetic fields during crystal growth. Nat. Commun. 2016, 7, 11555.

(4) Word Resources Institute (WRI). 2017, Climate Analysis Indicators Tool (CAIT) 2.0: WRI's climate data explorer. http://cait. wri.org (accessed June 20, 2018).

(5) United States Environmental Protection Agency (EPA). 2016, Climate change indicators in the United States: global greenhouse gas emissions. http://www.epa.gov/climate-indicators (accessed June 20, 2018).

(6) Braun, E.; Zurhelle, A. F.; Thijssen, W.; Schnell, S. K.; Lin, L.-C.; Kim, J.; Thompson, J. A.; Smit, B. High-throughput computational screening of nanoporous adsorbents for $\mathrm{CO} 2$ capture from natural gas. Mol. Syst. Des. Eng. 2016, 1, 175-188.

(7) Xiang, Z.; Mercado, R.; Huck, J. M.; Wang, H.; Guo, Z.; Wang, W.; Cao, D.; Haranczyk, M.; Smit, B. Systematic tuning and multifunctionalization of covalent organic polymers for enhanced carbon capture. J. Am. Chem. Soc. 2015, 137, 13301-13307.

(8) Liu, H.; Liu, B.; Lin, L.-C.; Chen, G.; Wu, Y.; Wang, J.; Gao, X.; Lv, Y.; Pan, Y.; Zhang, X.; et al. A hybrid absorption-adsorption method to efficiently capture carbon. Nat. Commun. 2014, 5, 5147.

(9) Lu, A.-H.; Hao, G.-P. Porous materials for carbon dioxide capture. Annu. Rep. Prog. Chem., Sect. A: Inorg. Chem. 2013, 109, 484503.

(10) Liu, Y.; Wilcox, J. Molecular Simulation Studies of CO2 Adsorption by Carbon Model Compounds for Carbon Capture and Sequestration Applications. Environ. Sci. Technol. 2013, 47, 95-101.

(11) Zhou, X.; Huang, W.; Miao, J.; Xia, Q.; Zhang, Z.; Wang, H.; Li, Z. Enhanced separation performance of a novel composite material Gro@MIL-101 for CO2/CH4 binary mixture. Chem. Eng. J. 2015, 266, 339-344.

(12) Srinivas, G.; Krungleviciute, V.; Guo, Z.-X.; Yildirim, T. Exceptional $\mathrm{CO} 2$ capture in a hierarchically porous carbon with simultaneous high surface area and pore volume. Energy Environ. Sci. 2014, 7, 335-342.

(13) Koenig, S. P.; Wang, L.; Pellegrino, J.; Bunch, J. S. Selective molecular sieving through porous graphene. Nat. Nanotechnol. 2012, 7, 728-732.

(14) Liu, H.; Dai, S.; Jiang, D.-e. Insights into CO2/N2 separation through nanoporous graphene from molecular dynamics. Nanoscale 2013, 5, 9984-9987.

(15) Du, H.; Li, J.; Zhang, J.; Su, G.; Li, X.; Zhao, Y. Separation of hydrogen and nitrogen gases with porous graphene membrane. J. Phys. Chem. C 2011, 115, 23261-23266.

(16) Tao, Y.; Xue, Q.; Liu, Z.; Shan, M.; Ling, C.; Wu, T.; Li, X. Tunable hydrogen separation in porous graphene membrane: firstprinciple and molecular dynamic simulation. ACS Appl. Mater. Interfaces 2014, 6, 8048-8058.

(17) Alaghemandi, M. Single layer hydrogenated graphyne membrane for selective hydrogen separation: a molecular dynamics simulation study. Chem. Phys. Lett. 2015, 629, 65-69.

(18) Meng, Z.; Zhang, X.; Zhang, Y.; Gao, H.; Wang, Y.; Shi, Q.; Rao, D.; Liu, Y.; Deng, K.; Lu, R. Graphdiyne as a high-efficiency membrane for separating oxygen from harmful gases: a first-principles study. ACS Appl. Mater. Interfaces 2016, 8, 28166-28170.

(19) Bartolomei, M.; Carmona-Novillo, E.; Hernández, M. I.; Campos-Martínez, J.; Pirani, F.; Giorgi, G.; Yamashita, K. Penetration Barrier of Water through Graphynes' Pores: First-Principles Predictions and Force Field Optimization. J. Phys. Chem. Lett. 2014, $5,751-755$.

(20) Ambrosetti, A.; Silvestrelli, P. L. Gas separation in nanoporous graphene from first principle calculations. J. Phys. Chem. C 2014, 118, 19172-19179.

(21) Wu, T.; Xue, Q.; Ling, C.; Shan, M.; Liu, Z.; Tao, Y.; Li, X. Fluorine-Modified Porous Graphene as Membrane for CO2/N2 Separation: Molecular Dynamic and First-Principles Simulations. J. Phys. Chem. C 2014, 118, 7369-7376.

(22) Zhu, T.; Ertekin, E. Phonon transport on two-dimensional graphene/boron nitride superlattices. Phys. Rev. B: Condens. Matter Mater. Phys. 2014, 90, 195209.

(23) Lin, S.; Buehler, M. J. Mechanics and molecular filtration performance of graphyne nanoweb membranes for selective water purification. Nanoscale 2013, 5, 11801.

(24) Baughman, R. H.; Eckhardt, H.; Kertesz, M. Structureproperty predictions for new planar forms of carbon: Layered phases containing sp2 and sp atoms. J. Chem. Phys. 1987, 87, 6687-6699.

(25) Li, G.; Li, Y.; Liu, H.; Guo, Y.; Li, Y.; Zhu, D. Architecture of graphdiyne nanoscale films. Chem. Commun. 2010, 46, 3256-3258.

(26) Bartolomei, M.; Giorgi, G. A novel nanoporous graphite based on graphynes: first-principles structure and carbon dioxide preferential physisorption. ACS Appl. Mater. Interfaces 2016, 8, 2799628003.

(27) Pirani, F.; Brizi, S.; Roncaratti, L. F.; Casavecchia, P.; Cappelletti, D.; Vecchiocattivi, F. Beyond the Lennard-Jones model: a simple and accurate potential function probed by high resolution 
scattering data useful for molecular dynamics simulations. Phys. Chem. Chem. Phys. 2008, 10, 5489-5503.

(28) Albernaz, A. F.; Aquilanti, V.; Barreto, P. R. P.; Caglioti, C.; Cruz, A. C. P. S.; Grossi, G.; Lombardi, A.; Palazzetti, F. Interactions of Hydrogen Molecules with Halogen-Containing Diatomics from $\mathrm{Ab}$ Initio Calculations: Spherical-Harmonics Representation and Characterization of the Intermolecular Potentials. J. Phys. Chem. A 2016, 120, 5315-5324.

(29) Yeamin, M. B.; Faginas-Lago, N.; Albertí, M.; Cuesta, I. G.; Sánchez-Marín, J.; Sánchez de Merás, A. M. J. Multi-scale theoretical investigation of molecular hydrogen adsorption over graphene: coronene as a case study. RSC Adv. 2014, 4, 54447-54453.

(30) Lago, N. F.; Larrañaga, F. H.; Albertí, M. On the suitability of the ILJ function to match different formulations of the electrostatic potential for water-water interactions. Eur. Phys. J. D 2009, 55, 75-85.

(31) Lago, N. F.; Albertí, M.; Lombardi, A.; Pirani, F. A force field for acetone: the transtition from small clusters to liquid phase investigated by molecular dynamics simulations. Theor. Chem. Acc. 2016, 135, 161.

(32) Faginas-Lago, N.; Yeamin, M. B.; Sánchez-Marin, J.; Cuesta, I. G.; Albertí, M.; de Merás, A. S. Modelization of the $\mathrm{H}_{2}$ adsorption on graphene and molecular dynamics simulation. Theor. Chem. Acc. 2017, 136, 91-99.

(33) Faginas-Lago, N.; Yeni, D.; Huarte, F.; Wang, Y.; Alcamí, M.; Martin, F. Adsorption of hydrogen molecules on carbon nanotubes using quantum chemistry and molecular dynamics. J. Phys. Chem. A 2016, 120, 6451-6458.

(34) Lombardi, A.; Faginas-Lago, N.; Pacifici, L.; Grossi, G. Energy transfer upon collision of selectively excited CO2 molecules: State-tostate cross sections and probabilities for modeling of atmospheres and gaseous flows. J. Chem. Phys. 2015, 143, 034307.

(35) Bartolomei, M.; Pirani, F.; Laganà, A.; Lombardi, A. A full dimensional grid empowered simulation of the $\mathrm{CO} 2+\mathrm{CO} 2$ processes. J. Comput. Chem. 2012, 33, 1806-1819.

(36) Lombardi, A.; Faginas-Lago, N.; Laganà, A.; Pirani, F.; Falcinelli, S. A bond-bond portable approach to intermolecular interactions: Simulations for N-methylacetamide and Carbon Dioxide Dimers. In Computational Science and its Applications-ICCSA 2012Lecture Notes in Computer Science; Murgante, B., et al. Eds, 2012; Vol 7333, pp 387-400.

(37) Falcinelli, S.; Rosi, M.; Candori, P.; Vecchiocattivi, F.; Bartocci, A.; Lombardi, A.; Faginas-Lago, N.; Pirani, F. Modeling the intermoleculr interactions and characterization of the dynamics of collisional autoionization processes. In Computational Science and its Applications-ICCSA 2013. ICCSA 2013. Lecture Notes in Computer Science; Murgante, B., et al. Eds, 2013; Vol 7971, pp 69-83.

(38) Lombardi, A.; Pirani, F.; Laganà, A.; Bartolomei, M. Energy transfer dynamics and kinetics of elementary processes (promoted) by gas-phase CO2-N2collisions: Selectivity control by the anisotropy of the interaction. J. Comput. Chem. 2016, 37, 1463-1475.

(39) Lombardi, A.; Faginas-Lago, N.; Gaia, G.; Federico, P.; Aquilanti, V. Collisional Energy Exchange in $\mathrm{CO} 2-\mathrm{N} 2$ Gaseous Mixtures. Computational Science and Its Applications. Lecture Notes in Computer Science, 2016; Part I, Vol. 9786, pp 246-257.

(40) Wilson, J.; Faginas-Lago, N.; Vekeman, J.; Cuesta, I. G.; Sánchez-Marín, J.; de Merás, A. S. Modeling the Interaction of Carbon Monoxide with Flexible Graphene: From Coupled Cluster Calculations to Molecular-Dynamics Simulations. ChemPhysChem 2018, 19, 774-783.

(41) Pitonák, M.; Heßelmann, A. Accurate intermolecular interaction energies from a combination of MP2 and TDDFT response theory. J. Chem. Theory Comput. 2010, 6, 168-178.

(42) Kendall, R. A.; Dunning, T. H.; Harrison, R. J. Electron affinities of the first-row atoms revisited. Systematic basis sets and wave functions. J. Chem. Phys. 1992, 96, 6796-6806.

(43) Werner, H.-J.; Knowles, P. J.; Knizia, G.; Manby, F. R.; Schütz, M. Molpro: a general-purpose quantum chemistry program package. Wiley Interdiscip. Rev.: Comput. Mol. Sci. 2012, 2, 242-253.
(44) Werner, H.-J.; Knowles, P. J.; Knizia, G.; Manby, F. R.; Schtz, M. MOLPRO, version 2015.1, A package of Ab Initio programs, 2015. http://www.molpro.net.

(45) Smith, W.; Yong, C. W.; Rodger, P. M. DL_POLY: application to molecular simulation. Mol. Simul. 2002, 28, 385-471.

(46) Pearlman, D. A.; Case, D. A.; Caldwell, J. W.; Ross, W. S.; Cheatham, T. E., III; DeBolt, S.; Ferguson, D.; Seibel, G.; Kollman, P. Amber, a package of computer programs for applying molecular mechanics, normal mode analysis, molecular dynamics and free energy calculations to simulate the structural and energetic properties of molecules. Comput. Phys. Commun. 1995, 91, 1-41.

(47) Cappelletti, D.; Pirani, F.; Bussery-Honvault, B.; Gomez, L.; Bartolomei, M. A bond-bond description of the intermolecular interaction energy: the case of weakly bound N2-H2 and N2-N2 complexes. Phys. Chem. Chem. Phys. 2008, 10, 4281-4293.

(48) Zhou, J.; Gao, X.; Liu, R.; Xie, Z.; Yang, J.; Zhang, S.; Zhang, G.; Liu, H.; Li, Y.; Zhang, J.; Liu, Z. Synthesis of graphdiyne nanowalls using acetylenic coupling reaction. J. Am. Chem. Soc. 2015, $137,7596-7599$.

(49) Elliott, J. R.; Lira, C. T. Introductory Chemical Engineering Thermodynamics, 2nd ed.; Prentice Hall: New Jersey, 2012.

(50) Ganesan, A.; Shaijumon, M. M. Activated graphene-derived porous carbon with exceptional gas adsorption properties. Microporous Mesoporous Mater. 2016, 220, 21-27.

(51) Ghosh, S.; Sevilla, M.; Fuertes, A. B.; Andreoli, E.; Ho, J.; Barron, A. R. Defining a performance map of porous carbon sorbents for high-pressure carbon dioxide uptake and carbon dioxide-methane selectivity. J. Mater. Chem. A 2016, 4, 14739-14751.

(52) Liu, B.; Smit, B. Comparative Molecular Simulation Study of $\mathrm{CO} 2 / \mathrm{N} 2$ and $\mathrm{CH} 4 / \mathrm{N} 2$ Separation in Zeolites and Metal-Organic Frameworks. Langmuir 2009, 25, 5918-5926. 